\title{
A Molecular Picture of the Adsorption of Ibuprofen and Benzoic Acid on hydrated amorphous Silica through DFT-D Calculations Combined with Solid State NMR Experiments
}

\author{
Frederik Tielens*, Nicolas Folliet, Louis Bondaz, Slavica Etemovic, \\ Florence Babonneau, Christel Gervais, and Thierry Azaïs
}

Sorbonne Universités, UPMC Univ Paris 06, CNRS, Collège de France, Laboratoire de Chimie de la Matière Condensée de Paris, 4 place Jussieu, 75005 Paris, France

*Corresponding author:

F. Tielens: frederik.tielens@upmc.fr 


\begin{abstract}
The presented research work is devoted to the investigation of the adsorption properties of biologically active molecules in mesoporous silicas. In particular, the interaction of ibuprofen with unique hydrated and functionalized silica carriers is unraveled by means of DFT-D calculations and experimental NMR experiments. The effects of temperature, degree of hydration, and adsorption site have been analyzed in detail to provide a molecular scale description of the adsorption and vectorization of a well-known drug, ibuprofen, on functionalized silicas. We are capable to conclude that the hydrated state, by the presence of a monolayer of water surface molecules on silica, plays the starring role.
\end{abstract}

Keywords: Silica, Ibuprofen, Benzoic Acid, NMR, DFT-D 


\section{Introduction}

During the last decade, the use of mesoporous silicas for the encapsulation and the controlled release of bioorganic molecules attracted a lot of interest ${ }^{1-7}$. The accessible pore volume of mesoporous silicas, such as MCM-41 silicas ${ }^{8}$, can store and release a large amount of different molecules including, organic solvents or biologically active molecules. ${ }^{9}$

In the field of medical applications, the molecular encapsulation of active pharmaceutical compounds allows a controlled release of biologically active molecules. For example, encapsulation of ibuprofen in mesoporous silica increases the solubility rate in physiological-like fluids. ${ }^{10}$ This property leads to the reduction of drug dosage and potential side-effects. A comprehension at the molecular level of the interactions involved in the adsorption phenomenon of ibuprofen onto the silica surface could lead to improve the conception of amorphous silica-based pharmaceutical vectors through a better control of the drug release over time ${ }^{11}$. Recent progress in structural studies of active pharmaceutical ingredients embedded in different drug carriers in the application of solidstate NMR spectroscopy has been reviewed by Skorupska et al. ${ }^{12}$

Ibuprofen release rate is related to the mesoporous silica's structural parameters and to the affinity between the molecule and the silica surface. The stronger the interactions the slower the release rate. ${ }^{3}$ In particular the functionalization of the pore surface with aminopropyl groups strongly affects the release kinetic. ${ }^{4}$ Moreover, the size and the geometry of the pores of textured mesoporous silicas also affect the release rate $^{3,5}$ as follows: small pores induce a slow release and thus a longer time of efficiency of the drug. However, while the release settings are well known experimentally, the theoretical characterization of the surface interactions between ibuprofen and the silica surface can still be improved. A very complete study by the group of Ugliengo ${ }^{13-14}$, presented a new realistic model for MCM-41, introducing hybrid functionals corrected for dispersion forces. They showed that electronic dispersion contributions to the interaction are critical for the correct description of the interactions. Recently, our group also built an MCM-41 DFT-D model and went one step further by investigating the important effect of hydration. ${ }^{15}$ Indeed silica is highly hydrophilic and the presence of water on the surface silanol groups has been proved to have an important effect on the adsorption complex's final molecular picture ${ }^{14}$, and thus also on the spectroscopic finger prints of the adsorption phenomenon on amorphous silica materials. 
In this paper we confront density functional calculations to solid state nuclear magnetic resonance (NMR) data in order to determine the origin and the strength of the interactions at the molecular level for ibuprofen and a small model molecule, benzoic acid, encapsulated in hydrated MCM-41 modified or not with organic moieties. To do so, different amorphous silica models were built: the influence of the presence of water on silica was first investigated, as well as the possible modification of the involved interactions following aminopropyl functionalization of the silica surface.

\section{Experimental Details}

\subsection{Material preparation}

MCM-41 is obtained by mixing under stirring at room temperature $\mathrm{H}_{2} \mathrm{O}, \mathrm{NaOH}$, cetyltrimethylammonium bromide and silica (Aerosil ${ }^{\circledR} 200$ ), in a given molar ratio of 20:0.25:0.1:1, respectively. The resulting white precipitated powder is filtered and plentifully washed with distilled water up to neutral $\mathrm{pH}$, then dried at $70^{\circ} \mathrm{C}$ for at least 48 hours. Samples are then calcined at $600^{\circ} \mathrm{C}$ for 6 hours under air flux to remove surfactant molecules. Functionalization of MCM-41 by aminopropyl groups was carried out through post-grafting synthesis by reacting $1 \mathrm{~g}$ of the mesoporous materials in an oven at $120^{\circ} \mathrm{C}$ for 2 hours with $1.33 \mathrm{~mL}$ of (3-Aminopropyl)triethoxysilane (APTES $\left(\mathrm{C}_{2} \mathrm{H}_{5} \mathrm{O}_{3} \mathrm{Si}\left(\mathrm{CH}_{2}\right)_{3} \mathrm{NH}_{2}\right)$ in $15 \mathrm{~mL}$ of acetonitrile under magnetic stirring. The mixture was stirred under argon overnight and the product was filtered, washed with a water/ethanol solution (50/50) and dried at $70^{\circ} \mathrm{C}$ overnight. APTES-functionalized mesoporous silica material is denoted as MCM-F in the forthcoming text.

The silica nanoparticles are obtained through the Stöber process: $5 \mathrm{~mL}$ of a solution of tetraethoxysilane (TEOS) in absolute ethanol $(0.90 \mathrm{M})$ is dropwise added to a solution of ammonia $(1,44 \mathrm{M})$ in a mixture of $46 \mathrm{~mL}$ of absolute ethanol and $10 \mathrm{~mL}$ of water. Vigorous stirring is maintained during 4 days during which time the solution turns turbid. After 4 days, the nanoparticles are washed by 4 cycles of centrifugation and dispersion in absolute ethanol. The average diameter is $360 \mathrm{~nm}$ and the BET surface area is $30 \mathrm{~m}^{2} \cdot \mathrm{g}^{-1}$ (Figure S1).

The loading of MCM-41 calcined materials and the adsorption on the Stöber silica particles was realized according to the incipient wetness procedure. The powders are loaded using a solution of ibuprofen, or benzoic acid in ethanol $\left(\sim 0.310 \mathrm{~mol} . \mathrm{L}^{-1}\right)$. Then, $0.500 \mathrm{~g}$ of powder is impregnated four times successively, with a small amount of the solution just allowing the powder to be wetted. The solvent is removed between two 
impregnations by evaporation at $70^{\circ} \mathrm{C}$ overnight. Afterwards, samples are quickly washed with $5 \mathrm{ml}$ of ethanol to remove the excess of non-encapsulated molecules. The control of this step is particularly crucial as an insufficient amount of ethanol can cause crystallization of the organic molecules in the samples, whereas an excess of ethanol leads to the partial release of the molecules. The MCM-41 (functionalized or not with APTES) samples loaded with ibuprofen and benzoic acid will be referred in the forthcoming text as MCM-Ibu/MCM-F-Ibu and MCM-BA/MCM-F-BA, respectively. Benzoic acid adsorbed Stöber silica particles will be refered as Stöber-BA in the forthcoming text.

\subsection{Solid-state NMR experiments}

${ }^{1} \mathrm{H}$ and ${ }^{13} \mathrm{C}$ solid state NMR experiments were performed on an AV300 Bruker spectrometer operating at $\Xi\left({ }^{1} \mathrm{H}\right)=300.13 \mathrm{MHz}$ and $\Xi\left({ }^{13} \mathrm{C}\right)=75.48 \mathrm{MHz} .4 \mathrm{~mm}$ zirconia rotors were spun at MAS frequency of 5 (for ${ }^{13} \mathrm{C}$ CP MAS spectra) or $14 \mathrm{kHz}$ (for ${ }^{13} \mathrm{C}$ MAS spectra). For ${ }^{1} \mathrm{H}$ MAS spectra, $2.5 \mathrm{~mm}$ zirconia rotors were used and spun at MAS frequency of $25 \mathrm{kHz}$. Recycle delay for ${ }^{1} \mathrm{H}$ and ${ }^{13} \mathrm{C}$ experiments was set to $2 \mathrm{~s}$. Spectra of functionalized samples were recorded using ${ }^{1} \mathrm{H}-{ }^{13} \mathrm{C}$ cross-polarisation (CP) experiments with proton decoupling (spinal-64) during acquisition. The chemical shift reference (0 ppm) for ${ }^{1} \mathrm{H}$ and ${ }^{13} \mathrm{C}$ was tetramethylsilane (TMS).

\subsection{Computational Details}

\subsubsection{Geometry optimizations and molecular dynamics calculations}

All calculations were performed using the $a b$ initio plane-wave pseudopotential approach as implemented in the VASP code ${ }^{16-17}$. The Perdew-Burke-Ernzerhof (PBE) functional ${ }^{18-}$ ${ }^{19}$ was chosen to perform the periodic DFT calculations with an accuracy on the overall convergence tested elsewhere ${ }^{20-25}$. The valence electrons were treated explicitly and their interactions with the ionic cores are described by the Projector Augmented-Wave method (PAW), ${ }^{26-27}$ which allows to use a low energy cut off equal to $400 \mathrm{eV}$ for the plane-wave basis. The integration over the Brillouin zone was performed on the $\Gamma$-point, in all calculations.

In the geometry optimizations at $0 \mathrm{~K}$, the positions of all atoms in the supercell are relaxed in the potential energy determined by the full quantum mechanical electronic structure until the total energy differences between the loops is less than $10^{-4} \mathrm{eV}$. In a second step, the Density Functional Theory - Dispersion (DFT-D) approach ${ }^{28}$ was applied as implemented in VASP, which consists in adding a semi-empirical dispersion potential to 
the conventional Kohn-Sham DFT energy. In particular we used the DFT-D method of Grimme $^{29}$ up to D2 correction and the vdW-DF2 functional of Langreth and Lundqvist. ${ }^{30}$ The potential energy surfaces (PES) were systematically explored by $a b$ initio molecular dynamics at $300 \mathrm{~K}$, starting from several configurations. The time step was set at $1.5 \mathrm{fs}$; several starting conformations were considered (but no statistic was performed on the starting conformations) and the run was stopped when an average stable conformation was reached after 500 fs or more; we used a micro-canonical ensemble in the NVE (constant number of molecules, constant volume and constant energy) approach. To avoid fluctuations due to the large time step chosen, the mass of hydrogen atom was set to 3 . The local minima found in the PES were then optimized at $0 \mathrm{~K}$. It is noticeable that this $a b$ initio molecular dynamics (MD) approach allowed us to find the minima of the PES showed in the present paper, which were not intuited beforehand.

\subsubsection{Calculation of interaction energies}

The energies of adsorption on the surface were calculated following different reactions: First, the neutral molecule (NM) was considered as the adsorbant. The adsorption energy $\Delta E_{\text {ads }}$ was calculated as follows:

$$
\Delta E_{\text {ads }}=E\left(\mathrm{NM}, \mathrm{Sil}, \mathrm{H}_{2} \mathrm{O}\right)-E(\mathrm{NM})-E(\mathrm{Sil})-\mathrm{n} \cdot E\left(\mathrm{H}_{2} \mathrm{O}\right)
$$

where $E(\mathrm{NM}), E(\mathrm{Sil})$, and $E\left(\mathrm{H}_{2} \mathrm{O}\right)$ are the total electronic energies of neutral molecule, silica surface, and one water molecule obtained after separate geometry optimization. $E\left(\mathrm{NM}, \mathrm{Sil}, \mathrm{H}_{2} \mathrm{O}\right)$ is the energy of the optimized $(\mathrm{NM}+$ silica + n.water $)$ system with the molecule adsorbed on the silica surface.

The adsorption energy can be decomposed into interaction and deformation energy, following:

$$
\Delta E_{\text {ads }}=\Delta E_{\text {int }}-\Delta E_{\text {deform-mol }}-\Delta E_{\text {deform-surface }}
$$

where $\Delta E_{\text {deform-mol }}$ and $\Delta E_{\text {deform-surface, being the energy required for a molecule or the }}$ surface, respectively, to adapt to the constraints induced by the surface or the molecule, respectively, and $\Delta E_{\text {int }}$ is the interaction energy between the surface and the molecule, in the geometries of the relaxed adsorption configuration. $\Delta E_{\text {ads }}$ is negative, for an exothermic process. 
The interaction energy of a dimer $\left(\Delta E_{\text {int.dimer }}\right)$ is calculated as follows:

$$
\Delta E_{\text {int.dimer }}=2 * \Delta E_{\text {int. } / \text { molec. }}=E_{\text {dimer }}-2 * E_{\text {molec. }}
$$

\subsubsection{Description of the pore surface models}

The adsorption of ibuprofen and benzoic acid was studied using a silica model structure described and characterized in a previous work. ${ }^{20}$ In summary the model consists of a $\mathrm{Si}_{27} \mathrm{O}_{54}$ slab hydrated with $13 \mathrm{H}_{2} \mathrm{O}$ molecules, forming 26 silanol groups. The silanol density at the surface is $5.8 \mathrm{~nm}^{-2}$, and unit cell dimensions are $12.77 \times 17.64 \times 25.17 \AA^{3}$. The slab has a density of approximately $1.7 \mathrm{~g} . \mathrm{cm}^{-3}$. The number of Si atoms belonging to exposed tetrahedra on the surface is approximately $8 \mathrm{~nm}^{-2}$. The same slab has been used to build model systems for catalyst supports and adsorption surfaces. ${ }^{11,15,37-50}$ Despite the fact that the global silanol density is higher than the one usually reported for MCM- $41^{51}$, locally the different expected types (terminal and geminal) are present on the model surface, enabling thus, a correct local description of the interaction sites. Bearing in mind that the proportion and type of silanol groups depend on the type of silica; as an example the geminal silanols represent $25 \%$ of all silanols for aerosil ${ }^{\circledR} 380$ silica while it is only $10 \%$ for a mesoporous silica MCM- $41^{9}$, the model is expected to be representative.

In this work we used the above silica model to investigate several combinations of $\mathrm{H}$ bonds between ibuprofen or benzoic acid and the silica surface model (bare silica surface). Moreover, the influence of a silanolate group ( $\left.\mathrm{SiO}^{-}\right)$was also investigated. In order to introduce this charged group, a proton was removed from one silanol and placed at the other side of the slab to form a $\mathrm{SiOH}_{2}{ }^{+}$group, i.e. keeping the electroneutrality of the periodic model. 


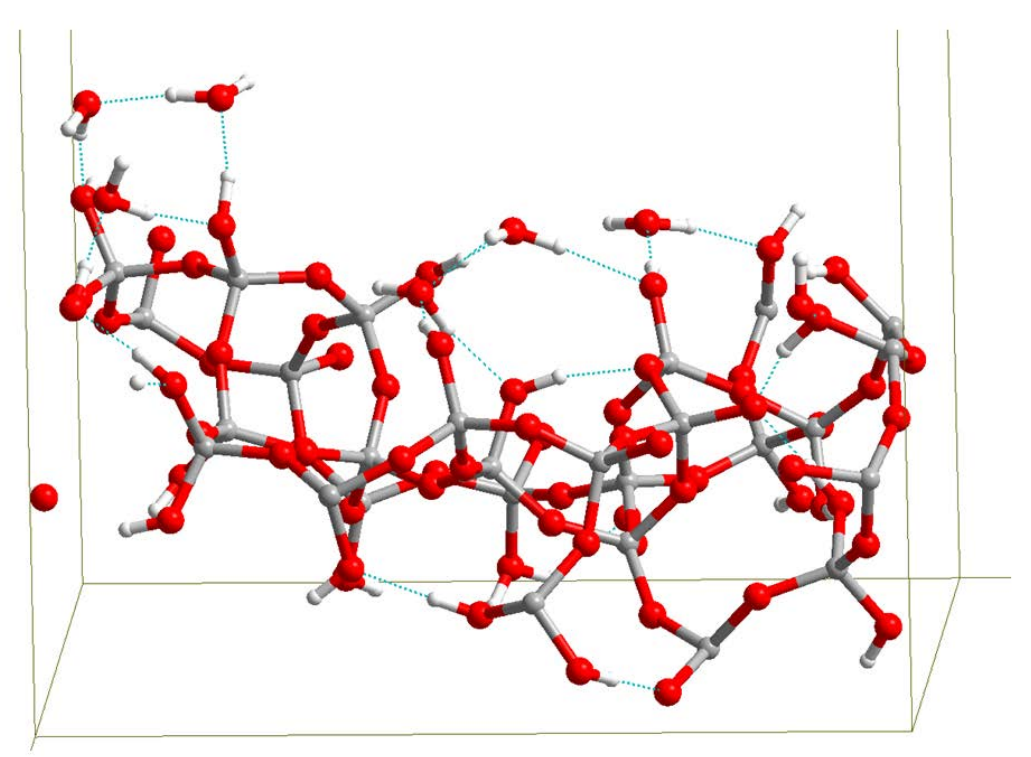

Figure 1. Microsolvated hydroxylated amorphous silica model used in the calculations. On every exposed silanol group a water molecule was added, i.e. 26 water molecules.

Finally, we also used the above model to study the effect of grafted aminopropyl groups on the adsorption properties of Ibu and BA. The functionalization of the silica is modeled by substitutions of OH-groups from surface silanols by an aminopropyl group ($\mathrm{C}_{3} \mathrm{H}_{6} \mathrm{NH}_{2}$ ) in order to obtain a final coverage of 3 chains per surface slab; i.e. $1.33 \mathrm{~nm}^{-2}$ which is coherent with experimental data $^{4}$.

\subsubsection{Ab initio calculations of NMR parameters}

The first principles NMR calculations were performed within Kohn-Sham DFT using the QUANTUM-ESPRESSO software (available online) ${ }^{31}$. The PBE generalized gradient approximation $^{18-19}$ was used and the valence electrons were described by norm conserving pseudopotentials ${ }^{32}$ in the Kleinman-Bylander ${ }^{33}$ form. The wave functions were expanded on a plane wave basis set with a kinetic energy cut-off of $1088 \mathrm{eV}$. The integral over the first Brillouin zone was performed using a Monkhorst-Pack $1 \times 1 \times 1 \mathrm{k}$ point grid for the charge density and chemical shift tensor calculation. The shielding tensor is computed using the GIPAW ${ }^{34}$ approach which permits the reproduction of the results of a fully converged all-electron calculation. Absolute shielding tensors are obtained and the ${ }^{13} \mathrm{C}$ scale was fixed so that experimental ${ }^{35}$ and calculated chemical shifts of all carbon sites in bulk ibuprofen ${ }^{36}$ coincide. 


\section{Results and discussion}

\subsection{Results}

\subsubsection{Interactions with the bare silica surface}

In order to investigate the dispersion of the investigated molecules on the silica surface, the interaction energy between two molecules of either benzoic acid (BA) or ibuprofen (Ibu) was first studied. The comparison of the interaction energy ( $\Delta E_{\text {int.dimer; }}$ Eq. 3) with the interaction with the bare silica surface, at a coverage of one molecule/slab $\left(=0.45 \mathrm{~nm}^{-}\right.$ ${ }^{2}$ ), provides a thermodynamic assessment of the driving force to segregate or to disperse on the substrate's surface. The starting conformation for the geometry optimization was chosen with a close (i.e. between $2-3 \AA$ ) proximity of the adsorbed molecule to the silica surface, to form a maximum number of hydrogen bonds between the molecule's carboxylic acid groups and the surface silanols. After geometry optimization, the highest adsorption energy $\mathrm{D} E_{\text {ads }}$, calculated using (eq. 1 ) with $\mathrm{n}=0$, for one BA molecule on the surface is $-0.64 \mathrm{eV}$ while for Ibu it is $-0.95 \mathrm{eV}$ (See Table 1). The adsorption energy is more favorable for Ibu in interaction with the surface than between two Ibu molecules (dimer formation), due to the formation of hydrogen bonds with the silica surface. In the case of BA the opposite is found.

In the case of Ibu a $\Delta E_{\text {ads. }}$ (eq. 2, See Table 1) between -0.8 to $-1.2 \mathrm{eV}$ is found, corresponding to the formation of four hydrogen bonds (typically $0.25 \mathrm{eV} / \mathrm{H}$-bond). Regarding the molecular dimers (in trans or cis conformation), an interaction energy of $0.8 \mathrm{eV}$ is calculated, being equivalent to a stabilization of $-0.4 \mathrm{eV}$ per molecule. A similar value is found for BA.

Table 1. Calculated interaction ( $\left.\Delta E_{\text {int }}\right)$ and adsorption energies ( $\left.\Delta E_{\text {ads }}\right)$ for the different investigated systems. Energies in $\mathrm{eV}$.

\begin{tabular}{|l|l|c|}
\hline & \multicolumn{1}{|c|}{ System } & $\Delta E_{\text {int }}$ \\
\hline Dimer & BA & -0.79 \\
& Ibu - cis dimer & -0.86 \\
& Ibu - trans dimer & -0.75 \\
\hline & System & $\Delta E_{\text {ads }}$ \\
\hline Silica surf. & BA & -0.64 \\
\hline
\end{tabular}




\begin{tabular}{|l|l|c|}
\hline & Ibu & -0.95 \\
\hline micro-solvated silica surf. & $\mathrm{H}_{2} \mathrm{O}$ & -0.67 \\
& BA & -0.25 \\
& Ibu & -0.38 \\
\hline Aminopropyl - & BA & 0.03 \\
functionalized silica surf. & & \\
& Ibu & 0.22 \\
\hline Aminopropyl - & BA & -1.09 \\
functionalized/micro- & Ibu & -1.27 \\
solvated silica surf. & & \\
\hline Silica surface $\quad$ with & & \\
silanolate & & -0.92 \\
Geminal Silanolate & BA & -0.69 \\
\hline \multirow{2}{*}{ Vicinal Silanolate } & Ibu & -1.75 \\
& BA & -1.71 \\
\hline & Ibu & -1.24 \\
& BA/ $\mu$ solvated & -1.40 \\
\hline
\end{tabular}

Overall one can conclude that Ibu is stabilized on the silica surface, compared with the formation of dimers in vacuum. The geometry optimization allows us to conclude that the bonds between the two studied molecules and the silica surface are strong enough to allow adsorption at $0 \mathrm{~K}$.

In order to investigate if the entropy does not compensate the adsorption trend at $0 \mathrm{~K}$, the effect of temperature was investigated using $a b$ initio molecular dynamics (AIMD) as implemented in the VASP code. The starting position of the molecules was the optimized adsorption geometry discussed above. At $300 \mathrm{~K}, \mathrm{BA}$ and Ibu were seen to detach from the surface during molecular dynamics. The thermal energy is thus sufficient to desorb the molecules.

\subsubsection{Interactions with a micro-solvated amorphous silica surface}


Some NMR experimental results indicate the micro-solvation of sol-gel silica surfaces ${ }^{11}$,

${ }^{47}$ through the presence of water molecules close to silanols. A silica surface was modeled accordingly, where a water molecule is positioned on each silanol group, i.e. microsolvated (See Fig. 1).

The position of BA and Ibu on the micro-solvated surface was optimized. Similarly to the bare surface, the adsorption energy between the molecules and the surface is calculated. The adsorption energy of the water molecules $\left(\Delta E_{\text {ads,H2O }}\right)$ of the micro-solvated system is defined as the difference between the hydrated silica surface ( $E_{\text {hydr.Surf }}$ ), the isolated water molecules $\left(E_{\mathrm{H} 2 \mathrm{O}}\right)$ and the (dehydrated) dry silica surface $\left(E_{\text {surf }}\right)$ :

$$
\Delta E_{\mathrm{ads}, \mathrm{H} 2 \mathrm{O}}=E_{\text {hydr.Surf }}-\mathrm{n} \cdot E_{\mathrm{H} 2 \mathrm{O}}-E_{\text {surf }}
$$

An adsorption energy of $-0.67 \mathrm{eV}$ is found. To interpret this value, one can compare it with the water/water energy of interaction. In water, each $\mathrm{H}_{2} \mathrm{O}$ molecule forms 2 - 3 hydrogen bonds at $273 \mathrm{~K}$, leading to thea stabilization energy for a water molecule in water estimated at -0.2 to $-0.3 \mathrm{eV}$ (experimental solvation energy $=-0.28 \mathrm{eV}^{52}$ ). Consequently, the water molecules have clearly a higher affinity for the silica surface than for other water molecules. This result can explain (i) the difficulty to remove entirely by conventional drying procedures water molecules from the surface of sol-gel silicas and (ii) why bioorganic molecules hardly displace these adsorbed water molecules ${ }^{11,47}$.

Adsorption energies for BA and Ibu on the microsolvated silica surface are calculated by the difference between the surface, the micro-solvated isolated molecule and the molecule on the micro-solvated surface. Each molecule is adsorbed via two water molecules on the surface (See Fig. 2).

The interaction energy is nevertheless much smaller than the adsorption energy calculated on the bare silica surface, a decrease of $0.39 \mathrm{eV}$ and $0.57 \mathrm{eV}$ is calculated for BA and for Ibu, respectively (See Table 1). Thus, the adsorption at $0 \mathrm{~K}$ seems to be less favorable than onto bare silica.

The same structures were then subjected to a MD calculation at $300 \mathrm{~K}$. In contrast to the bare surface, the molecules of BA and Ibu do not desorb immediately. The ibuprofen desorption phenomenon is much slower (observed after $0.3 \mathrm{ps}$ ), compared to the bare silica surface, where it was observed after a few fs. Indeed, the number of hydrogen bonds 
formed is higher in the case of micro-solvated surface thanks to the mediation from $\mathrm{H}_{2} \mathrm{O}$ molecules.

BA is found to be much more mobile than Ibu and can easily switch from silanol adsorption site on the micro-solvated surface. It desorbs and re-adsorbs thanks to the presence of water molecules

One can therefore predict that the adsorption/desorption of the organic molecules on a micro-solvated surface is dynamic; the molecules stay close to the surface and move along it. Moreover, the mobility of these molecules in all dimensions of space during the desorption phase is in agreement with the experimental NMR data, showing the mobility of the molecules in the mesoporous silicas as mentioned previously. ${ }^{53}$ Thus, the rapid reorientation of the molecules inside the porous network of mesoporous silica could be due to both a confinement effect and the specific dynamic phenomenon of adsorption/desorption induced by the interaction of the encapsulated BA or Ibu with water adsorbed molecules.

Thus, the micro-solvated silica surface is found to adsorb better BA and Ibu than the bare silica surface at $300 \mathrm{~K}$. The $\mathrm{H}_{2} \mathrm{O}$ layer can be compared to an adhesive layer between the organic molecules and the silica surface. The $\mathrm{H}_{2} \mathrm{O}$ molecules tend to stay close to the same silanol group throughout MD simulation, indicating a strong interaction with this group. Nevertheless, in some cases $\mathrm{H}_{2} \mathrm{O}$ molecules were found to desorb, and this only if a hydrogen bond is reformed, either with another organic molecule or with another desorbed $\mathrm{H}_{2} \mathrm{O}$ molecule. The low mobility of $\mathrm{H}_{2} \mathrm{O}$ molecules on the surface can be explained by the strength of the interaction between these molecules and the silanol groups and thus the difficulty for them to desorb efficiently. Overall, Ibu or BA adsorption is not strong enough to remove a $\mathrm{H}_{2} \mathrm{O}$ molecule from the silica surface. 

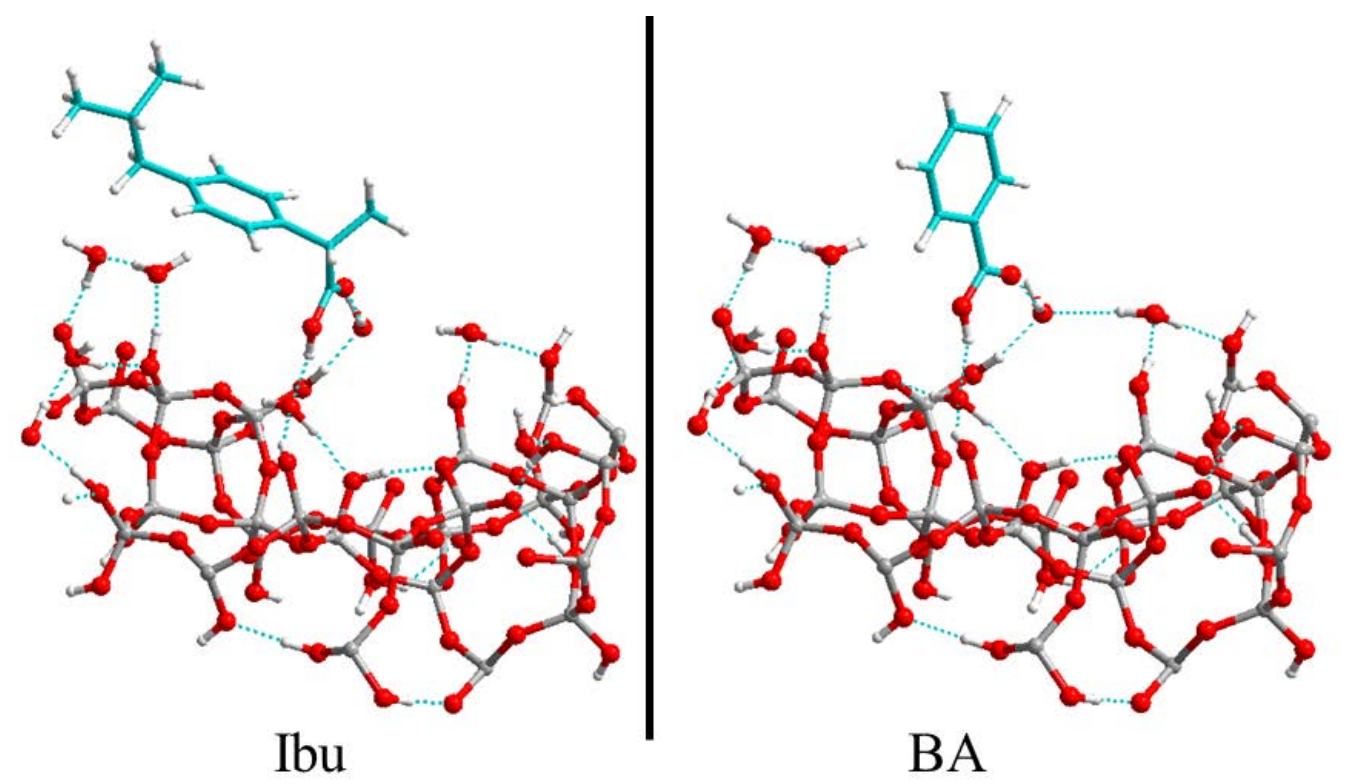

Figure 2. Optimized geometries of Ibu and BA on micro-solvated hydroxylated amorphous silica.

\subsubsection{Charged silica surface}

The study of the micro-solvated surface has allowed to highlight the central role of water in the adsorption of ibuprofen and benzoic acid in mesoporous silicas. The aim to study the different surfaces discussed above is to compare the influence of a strong interaction with the surface with the influence of microsolvation, as well as to simulate the effect of $\mathrm{pH}$ in an hydrated environment. The presence of a silanolate (Si- $\mathrm{O}^{-}$group) in geminal or vicinal position is also investigated. Considering a silanolate on the surface can be a local approach to simulate $\mathrm{pH}$ variation.

The interaction energies are calculated using eq. (1). In the case of the charged surface, it was found that the silanolates (both germinal and vicinal) show a strong interaction with the studied organic molecules (Table 1).

It should be noted that in our model the geminal silanolate was involved in a proton shift with a neighboring silanol group, which reorganizes its excess charge more efficiently by the formation of more hydrogen bonds.

These results allow confirming that interactions with silanolate are stronger than for a silanol.

Finally, a molecular dynamics simulation for a surface containing a silanolate was investigated. Placing the BA molecule on the vicinal silanolate adsorption site generated its deprotonation. BA remains adsorbed very strongly for a period of $0.6 \mathrm{ps}$, the H-bonds strength being due to the charge of the carboxylate group which is shared by $4 \mathrm{H}$-bonds 
with the vicinal silanols. It is observed that the carboxylate group moves very little contrary to the carbon-carbon bond between the carboxylate and the aromatic ring which shows a bending reflecting the strength of the H-bonds.

A low mobility of the carboxylic acid around the charged geminal site is observed. Proton exchange is observed during this process (a dozen hydrogen bonds give rise to proton exchange). These proton exchange interactions lead to a strong adsorption of BA throughout the molecular dynamics simulation.

In the case of Ibu adsorbed on a charged surface in vicinal position the same behavior as BA is observed. The proton of the carboxylic acid is exchanged with the silanolate and a very stable adsorbed carboxylate is formed.

If Ibu is placed in the neighborhood of a geminal silanolate, a very fast desorption is observed, due to the breaking/formation dynamics of the hydrogen bonding with the silanolate. However, after 0.1 ps the hydrogen bonding network does not resist desorption of the molecule and acts similarly to the dehydrated (dry) silica surface. From these results one can conclude that a very strong interaction does not necessarily keep an adsorbed molecule on the surface: unlike the micro-solvated surface, no dynamic mechanism similar to the action of $\mathrm{H}_{2} \mathrm{O}$ molecules allows a desorbed molecule to re-adsorb.

Therefore, it seems that the adsorption/desorption process of Ibu and BA molecules in mesoporous silicas is governed by entropy rather than by the formation of hydrogen bonds with the surface.

\subsubsection{Functionalized silica surface}

Another effect, which is expected to influence strongly the adsorption effects of silica is the functionalization of its surface by an aminopropyl group ( $\left.\mathrm{Si}-\mathrm{CH}_{2}-\mathrm{CH}_{2}-\mathrm{CH}_{2}-\mathrm{NH}_{2}\right)$. Aminopropyl functionalization is known to slow down the release of Ibu encapsulated in mesoporous silica. ${ }^{1}$ Looking at the results for the aminopropyl functionalized surface, an increase in interaction energy is found for BA and Ibu (See Table 1).

This is explained by the fact that the bare functionalized surface forms hydrogen bonds between the silanol groups and the amino groups by folding the aminopropyl carbon chains towards the surface (Figure 3). The absence of water molecules inhibits the interaction via H-bond formation between the amino groups of the aminopropyls and the organic molecules of interest. The H-bond network in both cases, i.e. dehydrated (or dry) and micro-solvated is shown in Figure 3. 


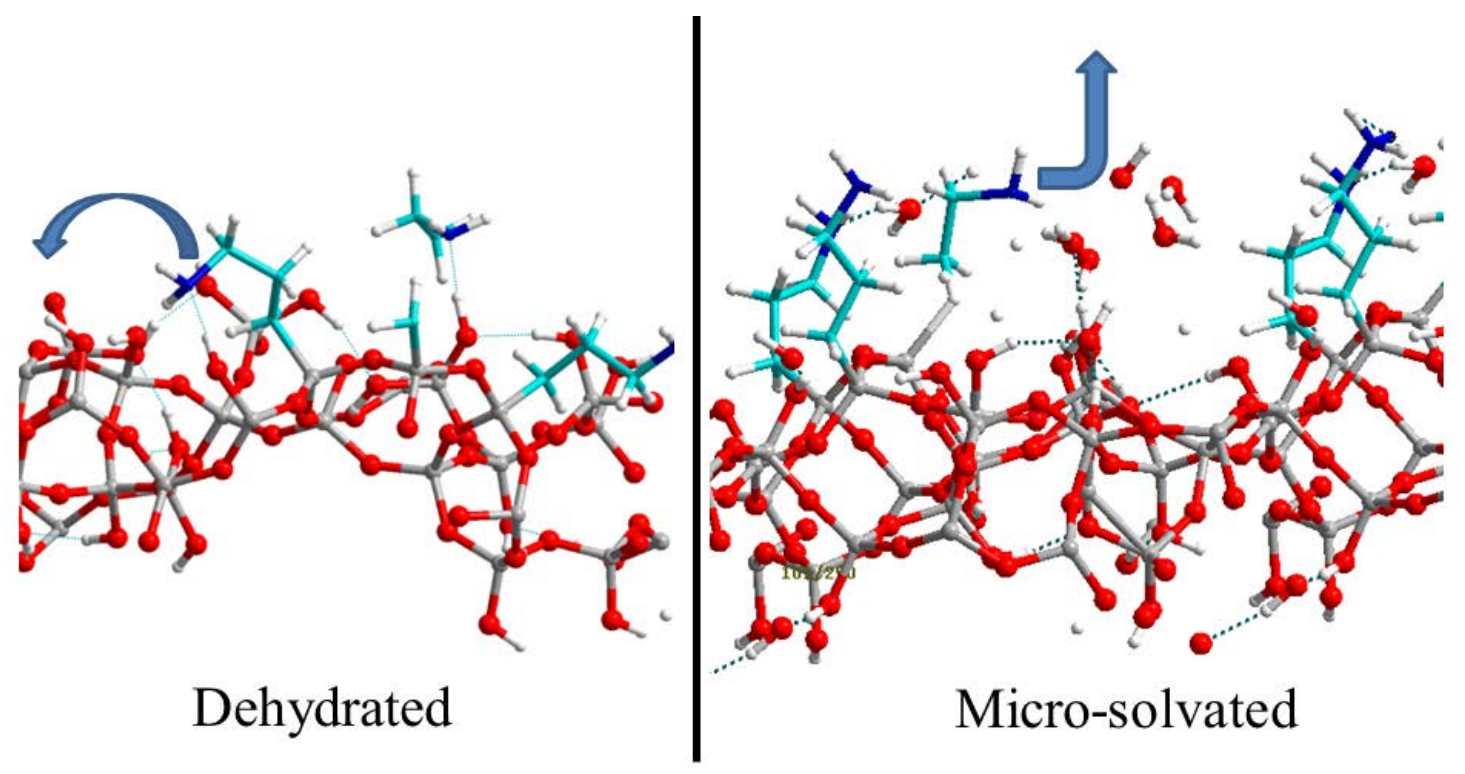

Figure 3. Models for the dehydrated or dry and micro-solvated hydroxylated functionalized amorphous silica surface, showing the effect of the water molecules on the orientation of the aminopropyl groups.

When adding an Ibu molecule to the surface, no interaction occurs with an amino or a silanol group since they are inaccessible due to the formation of an hydrogen bond between both functional groups, i.e. blocking the adsorption sites.

The extra stabilization obtained for the global system corresponds to the additional stabilization of an hydrogen bond between a silanol group and an amine group with respect to a carboxylic acid-amine hydrogen bonding. However, when the surface is micro-solvated by $\mathrm{H}_{2} \mathrm{O}$ molecules, the stabilization obtained by the adsorption of molecules is very large ( $-1.09 \mathrm{eV}$ for BA and $-1.27 \mathrm{eV}$ for Ibu). The adsorption energies obtained are larger than for the micro-solvated silica surface as well as for the bare silica surface. It should be noted that the interaction between the functionalized surface and Ibu occurs via H-bond between the amine group and the carboxylic acid group, without the presence of water molecules. Indeed, silanols are already stabilized by water molecules, the amine groups can thus interact with the organic molecules without destabilization in which the silanol loses hydrogen bonding with the amine (as in the case of the dehydrated functionalized surface). When observing the optimized structures, one can see that the aminopropyl groups allow the investigated organic molecules to interact more efficiently than on the bare surface by forming an hydrogen bond with both a water molecule and an amine group (See Figure 4). 


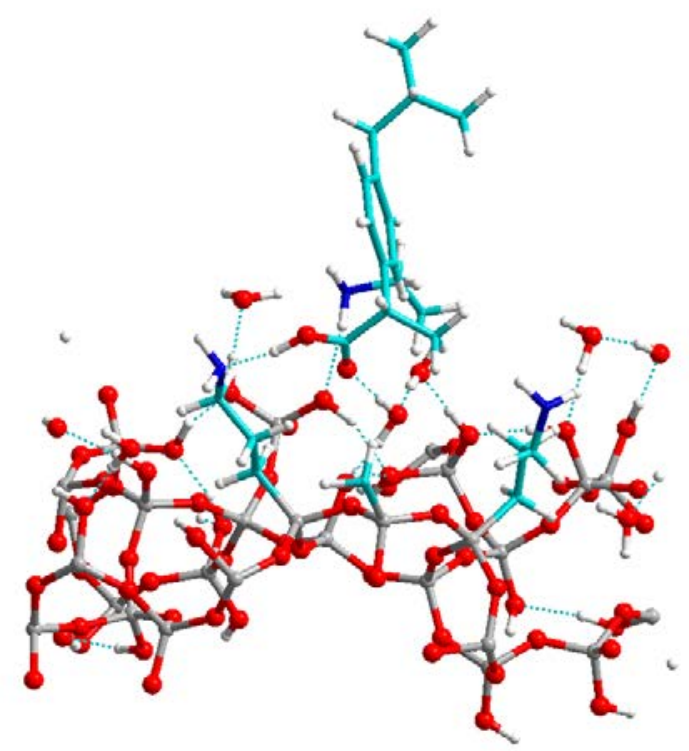

Figure 4. Optimized geometry for Ibu adsorbed on micro-solvated functionalized hydroxylated amorphous silica showing the H-bond formation between the aminopropyl amino groups and the carboxylic group of Ibu. The role of the water molecules as "glue" can also be observed.

Molecular dynamics on the functionalized surfaces by aminopropyl groups without micro-solvation confirms that the adsorption of organic molecules on this surface is very unfavorable. For both studied molecules, desorption occurs instantaneously.

From the molecular dynamics simulations, microsolvated silica surface functionalized with aminopropyl groups and Ibu or BA show a behavior similar to the non-functionalized micro-solvated surface. Indeed, the molecules of Ibu and BA are attached to the surface by forming hydrogen bonds between the carboxylic acid and the $\mathrm{H}_{2} \mathrm{O}$ molecules or amine groups. However, after 0.15 ps the molecules are desorbed and have no longer any interaction with the surface. We also note that aminopropyl groups naturally bend and reorient towards the surface silanols at $300 \mathrm{~K}$.

The global behavior of the functionalized surface is very similar to that of the bare surface when placed in the presence of studied molecules. Indeed, the silanol groups of the bare surface prefer to form intermolecular H-bonds between them, rather than with the molecule. This behavior is always observed for the functionalized surface together with the fact that aminopropyl groups fold towards the surface to form $\mathrm{H}$-bonds with the silanol groups. 
BA and Ibu remain adsorbed on the surface through H-bonds formed with amine groups or $\mathrm{H}_{2} \mathrm{O}$ molecules. Nevertheless it shows a relative high mobility.

Ibu interacts with a network of water molecules and amino groups, however the presence of aminopropyl has an impact on the mobility and adsorption strength. Although, the water molecules act as a glue, Ibu is very weakly adsorbed on microsolvated silica, the aminopropyl groups are able to fetch the almost desorbing molecule and bring it back in the hydrogen network formed by the water molecules and the aminopropyl groups. This behavior has an effect on the desorption kinetics, i.e. slower desorption which is in agreement with experiment. ${ }^{54}$

The aminopropyl functionalized surface was found to improve the adsorption properties of silica towards the organic molecules investigated. The stronger adsorption is an indirect result of the geometry and flexibility of the amino groups (See Table 1). Due to their structure which can unfold and fold under the influence of hydrogen bonds with water molecules they allow a higher adsorption than on the unfunctionalized surface.

\subsubsection{NMR experiments and calculations}

Experimentally, the weak interaction between Ibu and BA with the silica surface at ambient temperature $\left(\mathrm{T}_{\mathrm{amb}}\right)$ can be evidenced through solid state NMR. It was shown that the molecules exhibit a rapid reorientation inside the porous network when they are encapsulated in MCM-41. Such dynamics induce the average of the homonuclear ${ }^{1} \mathrm{H}-{ }^{1} \mathrm{H}$ interaction leading to highly resolved ${ }^{1} \mathrm{H}$ spectra in the solid state ${ }^{55}$ (Figure S2), and very poor efficiency of the ${ }^{1} \mathrm{H}-{ }^{13} \mathrm{C}$ cross polarization (CP) experiment ${ }^{56}$. This very specific highly mobile behavior is a consequence from both a physical confinement and the weak chemical affinity between Ibu and BA with the silica in good agreement AIMD at $300 \mathrm{~K}$ presented previously and showing that BA and Ibu molecules detach from the surface during molecular dynamics. This latter point can be investigated through the study of the adsorption of $\mathrm{AB}$ onto $\mathrm{SiO}_{2}$ spherical microparticles. This system allows to overcome confinement effect and to focus on the chemical affinity. The adsorption procedure was exactly the same than for MCM-41 (incipient wetness impregnation ${ }^{57}$ ) but the silica material was Stöber silica nanoparticles ${ }^{58}$. As a result, the amount of adsorbed molecules is rather low and correspond to a coverage of less than a single monolayer at the surface 
of the particles. The ${ }^{13} \mathrm{C}$ solid state NMR spectra of AB-Stöber-BA and MCM-BA samples reflect these features and are displayed in Fig. 5: whereas MCM-BA exhibits intense and narrow ${ }^{13} \mathrm{C}$ resonances characteristic of the high loading amount and the mobility of the molecules, in contrast Stöber-BA displays a weaker and broader signal corresponding to a lower amount of molecules.

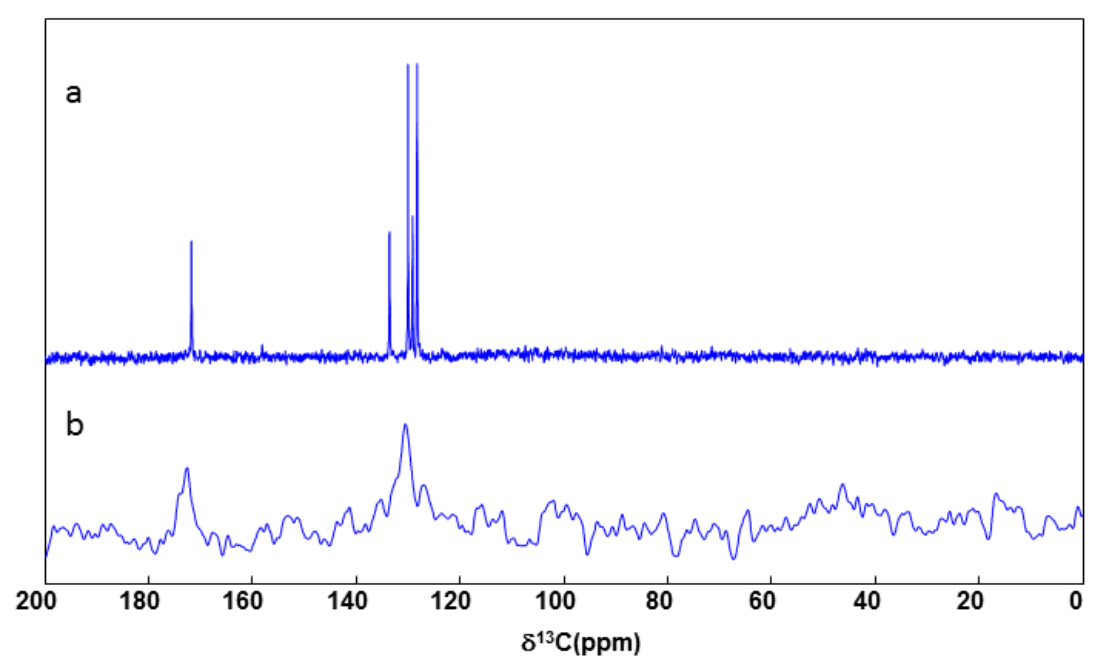

Figure 5. a) ${ }^{13} \mathrm{C}$ MAS NMR spectra of MCM-BA and b) ${ }^{13} \mathrm{C}$ CP MAS NMR spectra of Stöber-BA.

The NMR results for Stöber BA are found to be in agreement with the molecular model. From this result it is assumed that another element (e.g. confinement effect) comes into play to stabilize Ibu and BA inside the pores of the MCM-41.

The effect of functionalisation is presented in Figure 6 showing ${ }^{13} \mathrm{C}$ solid state NMR spectra of MCM-(F)-BA and MCM-(F)-Ibu samples. 

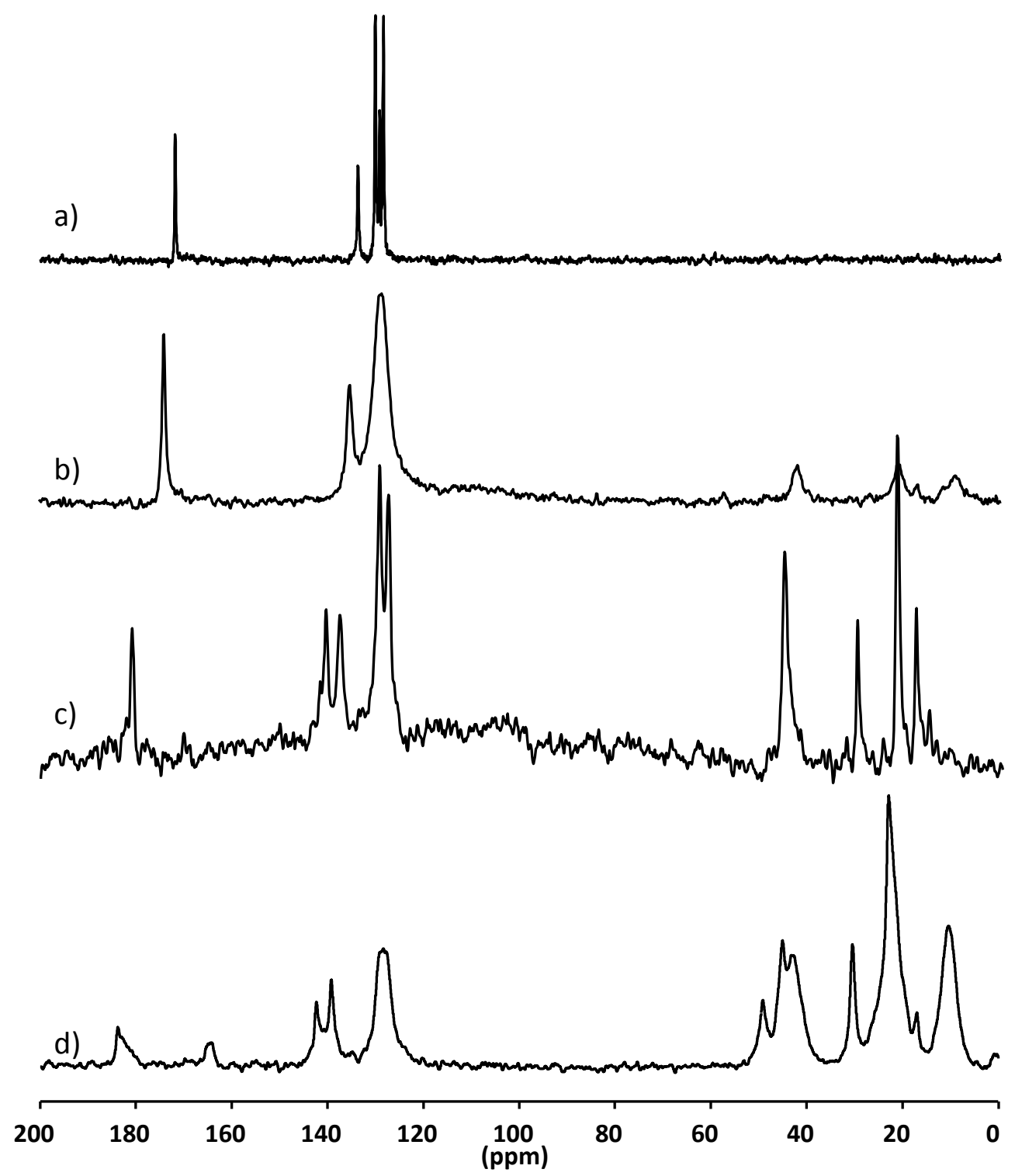

Figure 6. ${ }^{13} \mathrm{C}$ MAS NMR spectra of a) MCM-BA and c) MCM-Ibu. ${ }^{13} \mathrm{C}$ CP MAS NMR spectra of b) MCM-F-BA and d) MCM-F-Ibu.

In contrast with non-functionalized samples, solid state CP MAS technique is efficient for MCM-F samples, implying a stronger heteronuclear ${ }^{1} \mathrm{H}-{ }^{13} \mathrm{C}$ dipolar interaction due to a lower mobility of the molecules. This can be explained either by the smaller available space in the pores and/or by stronger interactions between the molecules and the surface. According to modeling, the micro-solvated aminopropyl functionalized surface is found to improve the adsorption properties of silica towards the organic molecules investigated, as an indirect result of the geometry and flexibility of the amine groups. Overall, compared to non-functionalized systems, MCM-F-BA/Ibu ${ }^{13} \mathrm{C}$ spectra show i) 3 
additional signals around 9.5, 21.5 and $42.5 \mathrm{ppm}$ corresponding to $\mathrm{Si}-\mathrm{CH}_{2}-\mathrm{CH}_{2}-\mathrm{CH}_{2}$ $\mathrm{NH}_{2}$ aminopropyl groups $\mathrm{ii}$ ) larger signals for $\mathrm{BA} / \mathrm{Ibu}$ signatures probably due to less mobility and/or wider distribution of chemical environments iii) a small shift towards higher ppm values for the position of the -COO carboxylic group (from 172 to $174 \mathrm{ppm}$ for BA and from 181 to $184 \mathrm{ppm}$ for Ibu).

This later point is interesting to be investigated in more details since it might be related to a change in the interactions between the molecules and the surface. Therefore, the calculated ${ }^{13} \mathrm{C}$ NMR chemical shift values of the COO groups in the different model are summarized in Table 2 (Full calculations are reported in SI, see Figures S3 - S8).

Table 2. Calculated ${ }^{13} \mathrm{C}$ chemical shift values of the COO groups for optimized geometries for BA and Ibu adsorbed on bare, micro-solvated, functionalized and microsolvated functionalized hydroxylated amorphous silica. (values in ppm)

\begin{tabular}{|c|l|l|l|l|l|l|l|l|}
\hline Model & MCM-BA & $\begin{array}{l}\text { MCM- } \\
\mu \mathrm{H}_{2} \mathrm{O}-\mathrm{BA}\end{array}$ & $\begin{array}{l}\text { MCM- } \\
\text { F-BA }\end{array}$ & $\begin{array}{l}\text { MCM-F- } \\
\mu \mathrm{H}_{2} \mathrm{O}-\mathrm{BA}\end{array}$ & $\begin{array}{c}\text { MCM- } \\
\text { Ibu }\end{array}$ & $\begin{array}{c}\text { MCM- } \\
\mu \mathrm{H}_{2} \mathrm{O}-\mathrm{BA}\end{array}$ & $\begin{array}{c}\text { MCM- } \\
\text { F-BA }\end{array}$ & $\begin{array}{c}\text { MCM-F- } \\
\mu \mathrm{H}_{2} \mathrm{O}-\mathrm{BA}\end{array}$ \\
\hline $\begin{array}{c}\delta\left({ }^{13} \mathrm{C}\right) \text { of } \\
\mathrm{COO}\end{array}$ & 173.2 & 171.4 & 171.5 & 173.9 & 181.2 & 181.8 & 178.7 & 182.9 \\
\hline
\end{tabular}

Interestingly, considering the microsolvated models which are possibly closer to experimental conditions, a shift of $+2.5 /+1.1 \mathrm{ppm}$ values is observed between MCM$\mu \mathrm{H}_{2} \mathrm{O}-\mathrm{BA} / \mathrm{Ibu}$ and MCM-F- $\mu \mathrm{H}_{2} \mathrm{O}-\mathrm{BA} / \mathrm{Ibu}$. It is difficult to be sure whether it is significant, but it is at least consistent with experiments.

\section{Conclusions}

We showed the importance of the presence of water on a silica surface. This monolayer which has been proved and predicted to be present on different silica systems, and in particular revealed in amino acid adsorption, plays the role of "glue” permitting a high mobility. Molecular dynamics confirmed these results once again here for the cases of ibuprofen and benzoic acid, which is in agreement with the experimental findings.

Concerning the functionalization of the silica surface with aminopropyl groups, the presence of water was shown to be crucial as well. The water molecules at the surface prevent the aminopropyl moiety to interact preferably with the surface silanols. The competition between water adsorption and interaction with the amino groups makes the aminopropyls available for interaction with ibuprofen or benzoic acid molecules. The 
functionalizing moieties act as "fishing line” grabbing the molecules dynamically, continuously making and breaking H-bonds.

These findings open the door to other similar functionalizations but with longer carbon chains to increase the flexibility or changing the amino group with an alcohol or introducing peptide bonds in the chains to generate a more rigid but well oriented selfassembly to "grab" the target molecules. By carefully choosing the functionalization compatible with the pore geometry of the mesoporous silica, one should be able to slow down or speed up the release rate of the molecule of interest. 


\section{Acknowledgements}

This work was performed using HPC resources from GENCI- [CCRT/CINES/IDRIS] (Grant 2015-[x2015082022]) and the CCRE of Université Pierre et Marie Curie.

\section{References}

1. Horcajada, P.; Ramila, A.; Ferey, G.; Vallet-Regi, M. Influence of Superficial Organic Modification of Mcm-41 Matrices on Drug Delivery Rate. Solid State Sciences 2006, 8, 1243-1249.

2. Horcajada, P.; Ramila, A.; Perez-Pariente, J.; Vallet-Regi, M. Influence of Pore Size of Mcm-41 Matrices on Drug Delivery Rate. Microporous and Mesoporous Materials 2004, 68, 105-109.

3. Horcajada, P.; Ramila, A.; Boulahya, K.; Gonzalez-Calbet, J.; Vallet-Regi, M. Bioactivity in Ordered Mesoporous Materials. Solid State Sciences 2004, 6, 1295-1300.

4. Munoz, B.; Ramila, A.; Perez-Pariente, J.; Diaz, I.; Vallet-Regi, M. Mcm-41 Organic Modification as Drug Delivery Rate Regulator. Chemistry of Materials 2003, 15 , 500-503.

5. Andersson, J.; Rosenholm, J.; Areva, S.; Linden, M. Influences of Material Characteristics on Ibuprofen Drug Loading and Release Profiles from Ordered Microand Mesoporous Silica Matrices. Chemistry of Materials 2004, 16, 4160-4167.

6. Izquierdo-Barba, I.; Martinez, A.; Doadrio, A. L.; Perez-Pariente, J.; Vallet-Regi, M. Release Evaluation of Drugs from Ordered Three-Dimensional Silica Structures. European Journal of Pharmaceutical Sciences 2005, 26, 365-373.

7. Vallet-Regi, M.; Ramila, A.; del Real, R. P.; Perez-Pariente, J. A New Property of Mcm-41: Drug Delivery System. Chemistry of Materials 2001, 13, 308-311.

8. Kresge, C. T.; Leonowicz, M. E.; Roth, W. J.; Vartuli, J. C.; Beck, J. S. Ordered Mesoporous Molecular-Sieves Synthesized by a Liquid-Crystal Template Mechanism. Nature 1992, 359, 710-712.

9. Rimola, A.; Costa, D.; Sodupe, M.; Lambert, J.-F.; Ugliengo, P. Silica Surface Features and Their Role in the Adsorption of Biomolecules: Computational Modeling and Experiments. Chemical Reviews 2013, 1134216.

10. Charnay, C.; Begu, S.; Tourne-Peteilh, C.; Nicole, L.; Lerner, D. A.; Devoisselle, J. M. Inclusion of Ibuprofen in Mesoporous Templated Silica: Drug Loading and Release Property. European Journal of Pharmaceutics and Biopharmaceutics 2004, 57, 533-540. 11. Folliet, N.; Gervais, C.; Costa, D.; Laurent, G.; Babonneau, F.; Stievano, L.; Lambert, J. F.; Tielens, F. A Molecular Picture of the Adsorption of Glycine in Mesoporous Silica through Nmr Experiments Combined with Dft-D Calculations. Journal of Physical Chemistry C 2013, 117, 4104-4114.

12. Skorupska, E.; Jeziorna, A.; Kazmierski, S.; Potrzebowski, M. J. Recent Progress in Solid-State Nmr Studies of Drugs Confined within Drug Delivery Systems. Solid State Nuclear Magnetic Resonance 2014, 57-58, 2-16.

13. Delle Piane, M.; Corno, M.; Pedone, A.; Dovesi, R.; Ugliengo, P. Large-Scale B3lyp Simulations of Ibuprofen Adsorbed in Mcm-41 Mesoporous Silica as Drug Delivery System. Journal of Physical Chemistry C 2014, 118, 26737-26749.

14. Delle Piane, M.; Vaccari, S.; Corno, M.; Ugliengo, P. Silica-Based Materials as Drug Adsorbents: First Principle Investigation on the Role of Water Microsolvation on Ibuprofen Adsorption. Journal of Physical Chemistry A 2014, 118, 5801-5807. 
15. Gierada, M.; Petit, I.; Handzlik, J.; Tielens, F. Hydration in Silica Based Mesoporous Materials: A DFT Model. Physical Chemistry Chemical Physics 2016, 18, 32962-32972.

16. Kresse, G.; Hafner, J. Ab-Initio Molecular-Dynamics for Liquid-Metals. Physical Review B 1993, 47, 558-561.

17. Kresse, G.; Hafner, J. Ab-Initio Molecular-Dynamics Simulation of the LiquidMetal Amorphous-Semiconductor Transition in Germanium. Physical Review B 1994, 49, 14251-14269.

18. Perdew, J. P.; Burke, K.; Ernzerhof, M. Generalized Gradient Approximation Made Simple. Physical Review Letters 1996, 77, 3865-3868.

19. Perdew, J. P.; Burke, K.; Ernzerhof, M. Generalized Gradient Approximation Made Simple (Vol 77, Pg 3865, 1996). Physical Review Letters 1997, 78, 1396-1396.

20. Tielens, F.; Gervais, C.; Lambert, J. F.; Mauri, F.; Costa, D. Ab Initio Study of the Hydroxylated Surface of Amorphous Silica: A Representative Model. Chemistry of Materials 2008, 20, 3336-3344.

21. Calatayud, M.; Tielens, F.; De Proft, F. Reactivity of Gas-Phase, Crystal and Supported V2o5 Systems Studied Using Density Functional Theory Based Reactivity Indices. Chemical Physics Letters 2008, 456, 59-63.

22. de Bocarme, T. V.; Chau, T. D.; Tielens, F.; Andres, J.; Gaspard, P.; Wang, R. L. C.; Kreuzer, H. J.; Kruse, N. Oxygen Adsorption on Gold Nanofacets and Model Clusters. Journal of Chemical Physics 2006, 125.

23. Tielens, F.; Andres, J.; Chau, T. D.; de Bocarme, T. V.; Kruse, N.; Geerlings, P. Molecular Oxygen Adsorption on Electropositive Nano Gold Tips. Chemical Physics Letters 2006, 421, 433-438.

24. Tielens, F.; Calatayud, M.; Dzwigaj, S.; Che, M. What Do Vanadium Framework Sites Look Like in Redox Model Silicate Zeolites? Microporous and Mesoporous Materials 2009, 119, 137-143.

25. Tielens, F.; Trejda, M.; Ziolek, M.; Dzwigaj, S. Nature of Vanadium Species in V Substituted Zeolites: A Combined Experimental and Theoretical Study. Catalysis Today 2008, 139, 221-226.

26. Blochl, P. E.; Jepsen, O.; Andersen, O. K. Improved Tetrahedron Method for Brillouin-Zone Integrations. Physical Review B 1994, 49, 16223-16233.

27. Kresse, G.; Joubert, D. From Ultrasoft Pseudopotentials to the Projector Augmented-Wave Method. Physical Review B 1999, 59, 1758-1775.

28. Wu, X.; Vargas, M. C.; Nayak, S.; Lotrich, V.; Scoles, G. Towards Extending the Applicability of Density Functional Theory to Weakly Bound Systems. Journal of Chemical Physics 2001, 115, 8748-8757.

29. Grimme, S. Semiempirical Gga-Type Density Functional Constructed with a Long-Range Dispersion Correction. Journal of Computational Chemistry 2006, 27, 17871799.

30. Lee, K.; Murray, E. D.; Kong, L.; Lundqvist, B. I.; Langreth, D. C. HigherAccuracy Van Der Waals Density Functional. Physical Review B 2010, 82.

31. Giannozzi, P.; Baroni, S.; Bonini, N.; Calandra, M.; Car, R.; Cavazzoni, C.; Ceresoli, D.; Chiarotti, G. L.; Cococcioni, M.; Dabo, I.; Dal Corso, A.; de Gironcoli, S.; Fabris, S.; Fratesi, G.; Gebauer, R.; Gerstmann, U.; Gougoussis, C.; Kokalj, A.; Lazzeri, M.; Martin-Samos, L.; Marzari, N.; Mauri, F.; Mazzarello, R.; Paolini, S.; Pasquarello, A.; Paulatto, L.; Sbraccia, C.; Scandolo, S.; Sclauzero, G.; Seitsonen, A. P.; Smogunov, A.; Umari, P.; Wentzcovitch, R. M. Quantum Espresso: A Modular and Open-Source Software Project for Quantum Simulations of Materials. Journal of Physics-Condensed Matter 2009, 21, 395502. 
32. Troullier, N.; Martins, J. L. Efficient Pseudopotentials for Plane-Wave Calculations. Physical Review B 1991, 43, 1993-2006.

33. Kleinman, L.; Bylander, D. M. Efficacious Form for Model Pseudopotentials. Physical Review Letters 1982, 48, 1425-1428.

34. Pickard, C. J.; Mauri, F. All-Electron Magnetic Response with Pseudopotentials: Nmr Chemical Shifts. Physical Review B 2001, 63, 245101.

35. Babonneau, F.; Yeung, L.; Steunou, N.; Gervais, C.; Ramila, A.; Vallet-Regi, M. Solid State Nmr Characterisation of Encapsulated Molecules in Mesoporous Silica. Journal of Sol-Gel Science and Technology 2004, 31, 219-223.

36. Shankland, N.; Wilson, C. C.; Florence, A. J.; Cox, P. J. Refinement of Ibuprofen at $100 \mathrm{~K}$ by Single-Crystal Pulsed Neutron Diffraction. Acta Crystallographica Section C-Crystal Structure Communications 1997, 53, 951-954.

37. Handzlik, J.; Grybos, R.; Tielens, F. Isolated Chromium(Vi) Oxide Species Supported on Al-Modified Silica: A Molecular Description. Journal of Physical Chemistry C 2016, 120, 17594-17603.

38. Guesmi, H.; Grybos, R.; Handzlik, J.; Tielens, F. Characterization of Tungsten Monomeric Oxide Species Supported on Hydroxylated Silica; a Dft Study. Rsc Advances 2016, 6, 39424-39432.

39. Gierada, M.; Michorczyk, P.; Tielens, F.; Handzlik, J. Reduction of ChromiaSilica Catalysts: A Molecular Picture. Journal of Catalysis 2016, 340, 122-135.

40. Tranca, D. C.; Wojtaszek-Gurdak, A.; Ziolek, M.; Tielens, F. Supported and Inserted Monomeric Niobium Oxide Species on/in Silica: A Molecular Picture. Physical Chemistry Chemical Physics 2015, 17, $22402-22411$.

41. Pfeiffer-Laplaud, M.; Costa, D.; Tielens, F.; Gaigeot, M.-P.; Sulpizi, M. Bimodal Acidity at the Amorphous Silica/Water Interface. Journal of physical Chemistry C 2015, 119, 27354-27362.

42. Neouze, M. A.; Kronstein, M.; Litschauer, M.; Puchberger, M.; Coelho, C.; Bonhomme, C.; Gervais, C.; Tielens, F. Exploring the Molecular Structure of Imidazolium-Silica-Based Nanoparticle Networks by Combining Solid-State Nmr Spectroscopy and First-Principles Calculations. Chemistry-a European Journal 2014, 20, 15188-15196.

43. Guesmi, H.; Gryboś, R.; Handzlik, J.; Tielens, F. Characterization of Molybdenum Monomeric Oxide Species Supported on Hydroxylated Silica; a Dft Study. Physical Chemistry Chemical Physics 2014, 16, 18253-18260.

44. Cimas, A.; Tielens, F.; Sulpizi, M.; Gaigeot, M. P.; Costa, D. The Amorphous Silica-Liquid Water Interface Studied by Ab Initio Molecular Dynamics (Aimd): Local Organization in Global Disorder. Journal of Physics-Condensed Matter 2014, 26, 244106.

45. Handzlik, J.; Grybos, R.; Tielens, F. Structure of Monomeric Chromium(Vi) Oxide Species Supported on Silica: Periodic and Cluster Dft Studies. Journal of Physical Chemistry C 2013, 117, 8138-8149.

46. Guesmi, H.; Tielens, F. Chromium Oxide Species Supported on Silica: A Representative Periodic Dft Model. Journal of Physical Chemistry C 2012, 116, 9941001.

47. Folliet, N.; Roiland, C.; Begu, S.; Aubert, A.; Mineva, T.; Goursot, A.; Selvaraj, K.; Duma, L.; Tielens, F.; Mauri, F.; Laurent, G.; Bonhomme, C.; Gervais, C.; Babonneau, F.; Azais, T. Investigation of the Interface in Silica-Encapsulated Liposomes by Combining Solid State Nmr and First Principles Calculations. Journal of the American Chemical Society 2011, 133, 16815-16827. 
48. Wojtaszek, A.; Sobczak, I.; Ziolek, M.; Tielens, F. The Formation of Gold Clusters Supported on Mesoporous Silica Material Surfaces: A Molecular Picture. Journal of Physical Chemistry C 2010, 114, 9002-9007.

49. Wojtaszek, A.; Sobczak, I.; Ziolek, M.; Tielens, F. Gold Grafted to Mesoporous Silica Surfaces, a Molecular Picture. Journal of Physical Chemistry C 2009, 113, 1385513859.

50. Islam, M. M.; Costa, D.; Calatayud, M.; Tielens, F. Characterization of Supported Vanadium Oxide Species on Silica: A Periodic Dft Investigation. Journal of Physical Chemistry C 2009, 113, 10740-10746.

51. Shenderovich, I. G.; Buntkowsky, G.; Schreiber, A.; Gedat, E.; Sharif, S.; Albrecht, J.; Golubev, N. S.; Findenegg, G. H.; Limbach, H. H. Pyridine-N-15 - a Mobile Nmr Sensor for Surface Acidity and Surface Defects of Mesoporous Silica. Journal of Physical Chemistry B 2003, 107, 11924-11939.

52. Chemistry Webbook, Nist Standard Reference Database Number 69, Release June 2005.

53. Babonneau, F.; Baccile, N.; Laurent, G.; Maquet, J.; Azais, T.; Gervais, C.; Bonhomme, C. Solid-State Nuclear Magnetic Resonance: A Valuable Tool to Explore Organic-Inorganic Interfaces in Silica-Based Hybrid Materials. Comptes Rendus Chimie 2010, 13, 58-68.

54. Contessotto, L.; Ghedini, E.; Pinna, F.; Signoretto, M.; Cerrato, G.; Crocella, V. Hybrid Organic-Inorganic Silica Gel Carriers with Controlled Drug-Delivery Properties. Chemistry-a European Journal 2009, 15, 12043-12049.

55. Aiello, D.; Folliet, N.; Laurent, G.; Testa, F.; Gervais, C.; Babonneau, F.; Azais, T. Solid State Nmr Characterization of Phenylphosphonic Acid Encapsulated in Sba-15 and Aminopropyl-Modified Sba-15. Microporous and Mesoporous Materials 2013, 166, 109-116.

56. Azais, T.; Hartmeyer, G.; Quignard, S.; Laurent, G.; Babonneau, F. Solution State Nmr Techniques Applied to Solid State Samples: Characterization of Benzoic Acid Confined in Mcm-41. Journal of Physical Chemistry C 2010, 114, 8884-8891.

57. Azais, T.; Hartmeyer, G.; Quignard, S.; Laurent, G.; Tourne-Peteilh, C.; Devoisselle, J. M.; Babonneau, F. Solid-State Nmr Characterization of Drug-Model Molecules Encapsulated in Mcm-41 Silica. Pure and Applied Chemistry 2009, 81, 13451355.

58. Azais, T.; Tourne-Peteilh, C.; Aussenac, F.; Baccile, N.; Coelho, C.; Devoisselle, J. M.; Babonneau, F. Solid-State Nmr Study of Ibuprofen Confined in Mcm-41 Material. Chemistry of Materials 2006, 18, 6382-6390. 
TOC Figure

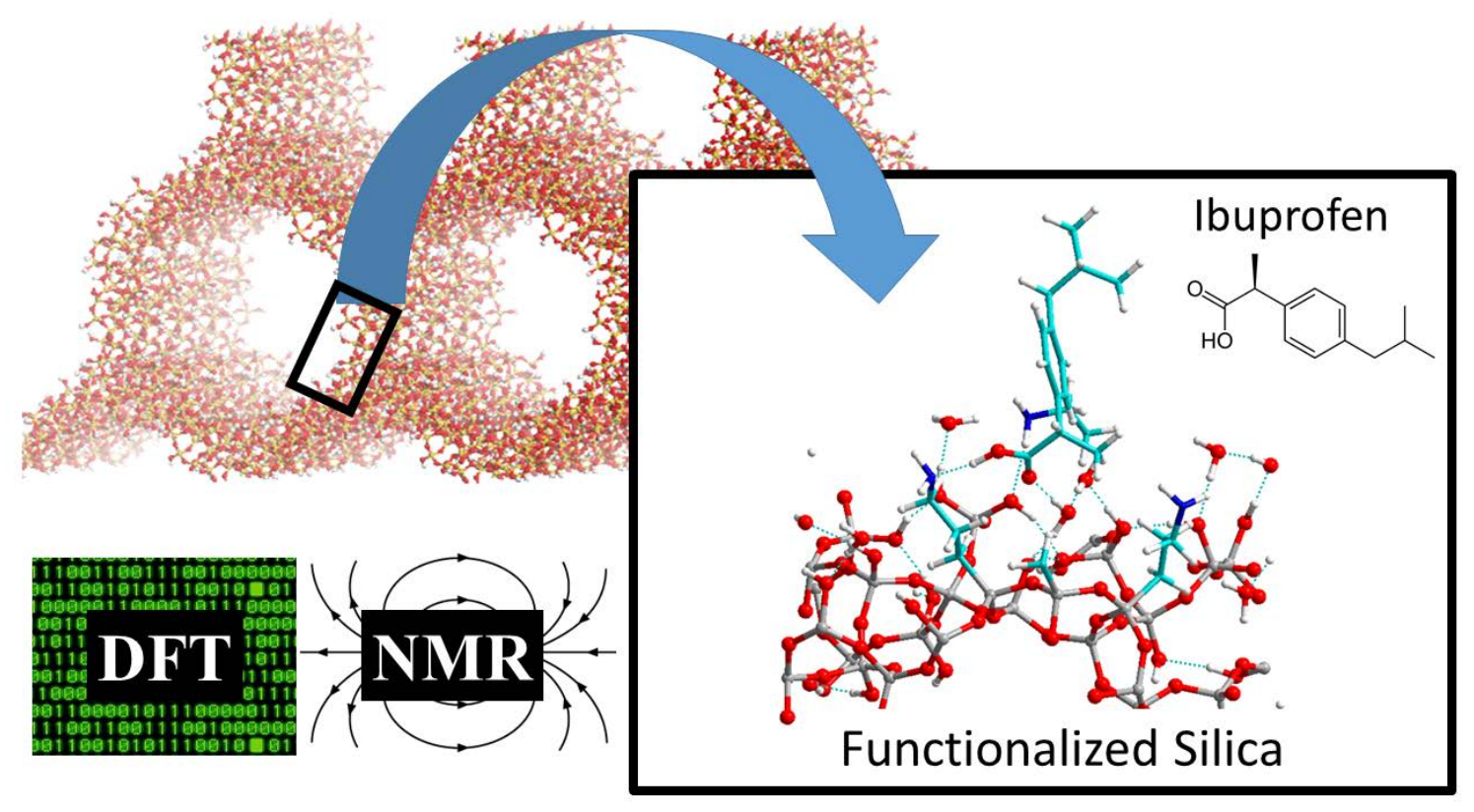




\title{
A Molecular Picture of the Adsorption of Ibuprofen and Benzoic Acid on hydrated amorphous Silica through DFT-D Calculations Combined with solid state NMR Experiments
}

\author{
Frederik Tielens ${ }^{*}$, Nicolas Folliet, Louis Bondaz, Slavica Etemovic, Florence \\ Babonneau, Christel Gervais, and Thierry Azaïs
}

Sorbonne Universités, UPMC Univ Paris 06, CNRS, Collège de France, Laboratoire de Chimie de la Matière Condensée de Paris, 4 place Jussieu, 75005 Paris, France

Supporting Information Available 
Figure S1: (a) Nitrogen adsorption/desorption isotherms and (b) SEM micrograph of silica Stöber nanoparticles. The BET surface area is $30 \mathrm{~m}^{2} \cdot \mathrm{g}^{-1}$ and the average diameter is $360 \mathrm{~nm}$.

(a)

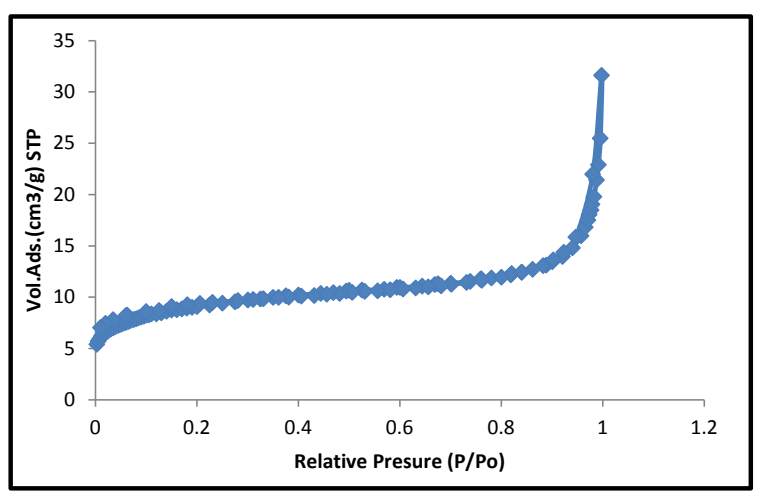

(b)

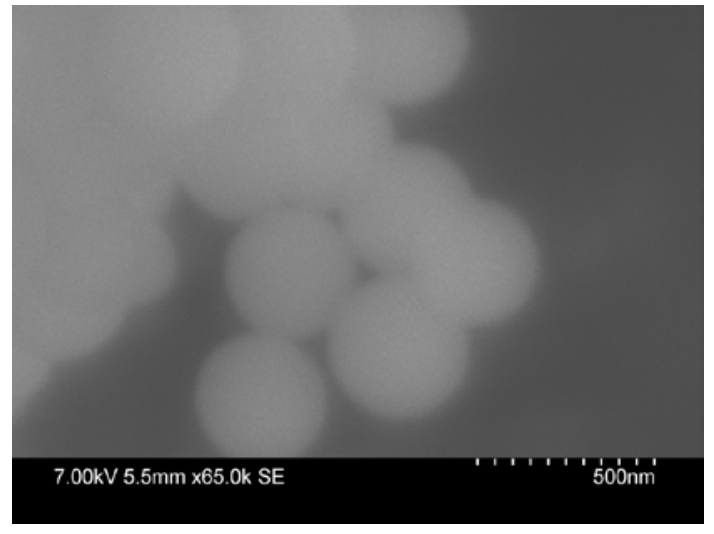


Figure S2: ${ }^{1} \mathrm{H}$ NMR spectra of (a) MCM-Ibu and (b) and MCM-BA with their respective assignment. * denotes adsorbed water

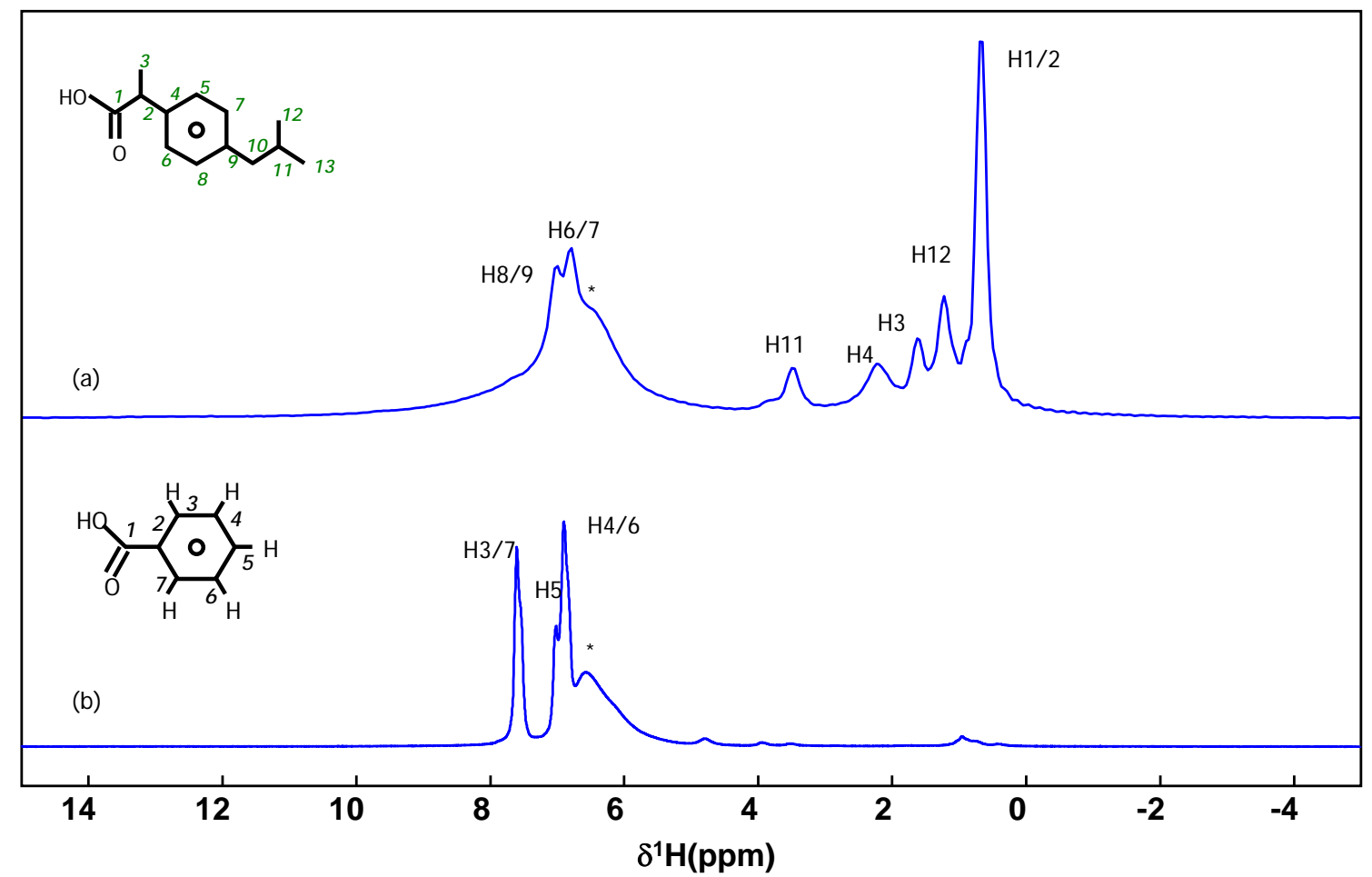


Table S3: Calculated ${ }^{13} \mathrm{C}$ and ${ }^{1} \mathrm{H}$ NMR chemical shift values for optimized geometries for BA adsorbed on bare or micro-solvated hydroxylated amorphous silica

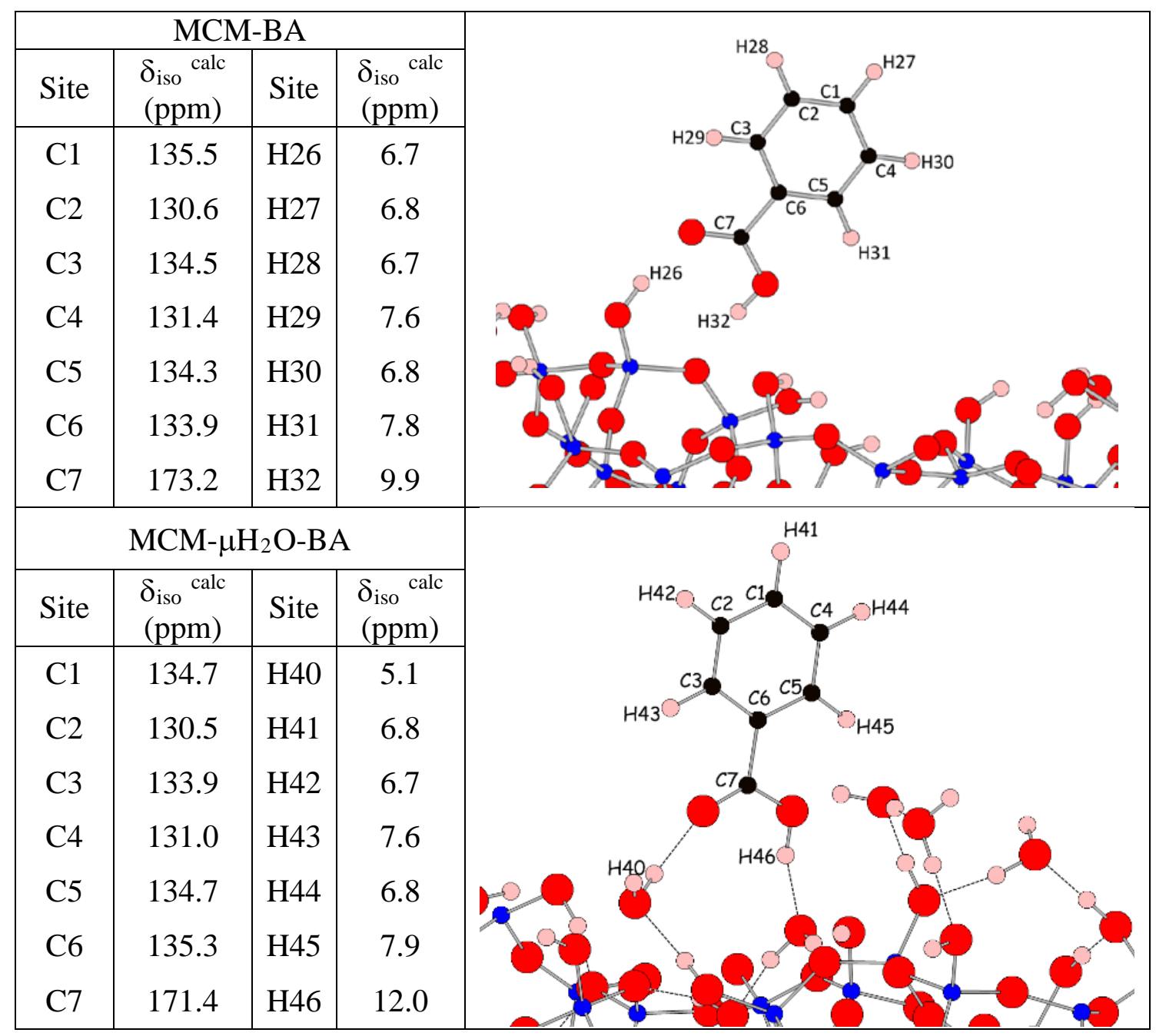


Table S4: Calculated ${ }^{13} \mathrm{C}$ and ${ }^{1} \mathrm{H}$ NMR chemical shift values for optimized geometries for BA adsorbed on bare or micro-solvated functionalized hydroxylated amorphous silica

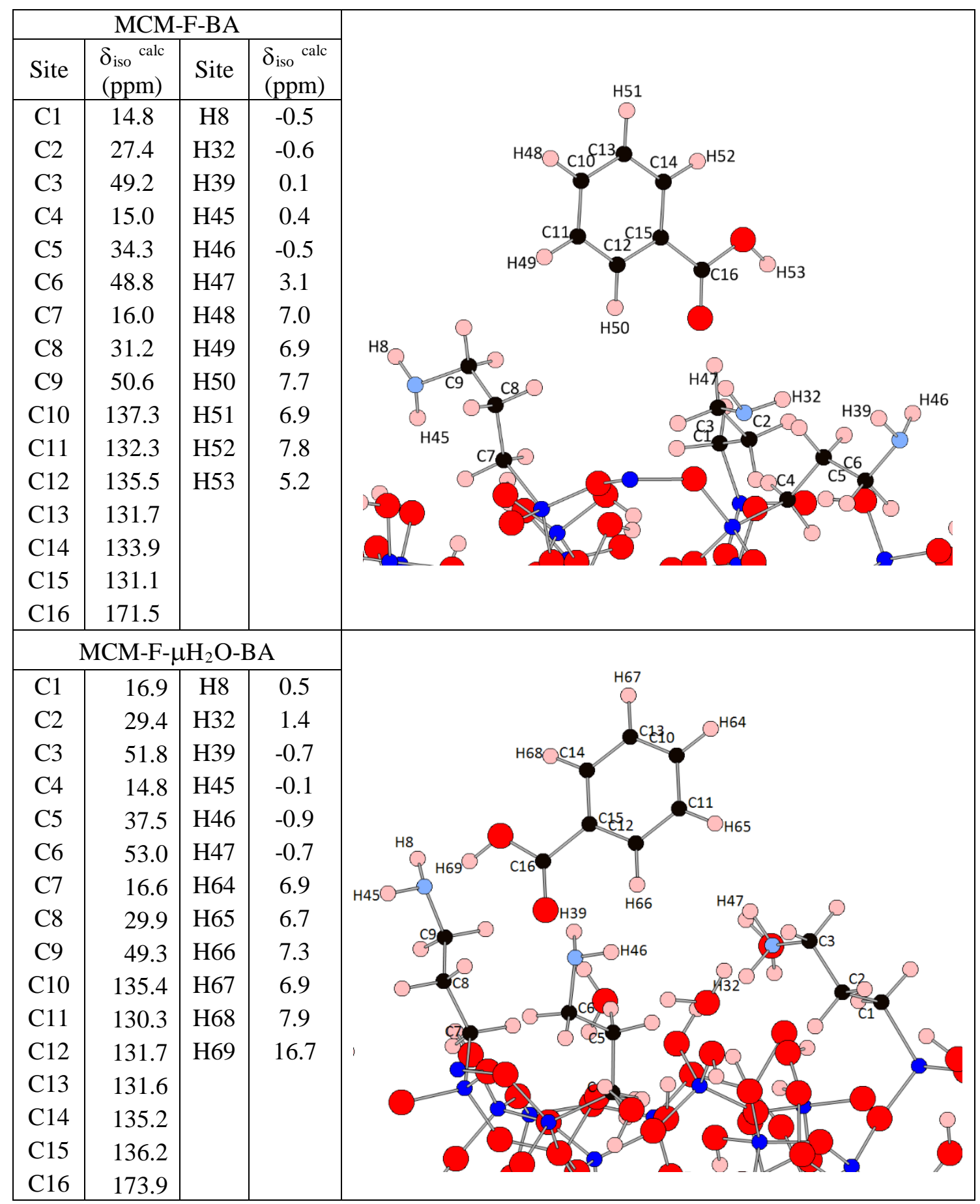


Table S5: Calculated ${ }^{13} \mathrm{C}$ and ${ }^{1} \mathrm{H}$ NMR chemical shift values for optimized geometries for Ibu adsorbed on bare hydroxylated amorphous silica

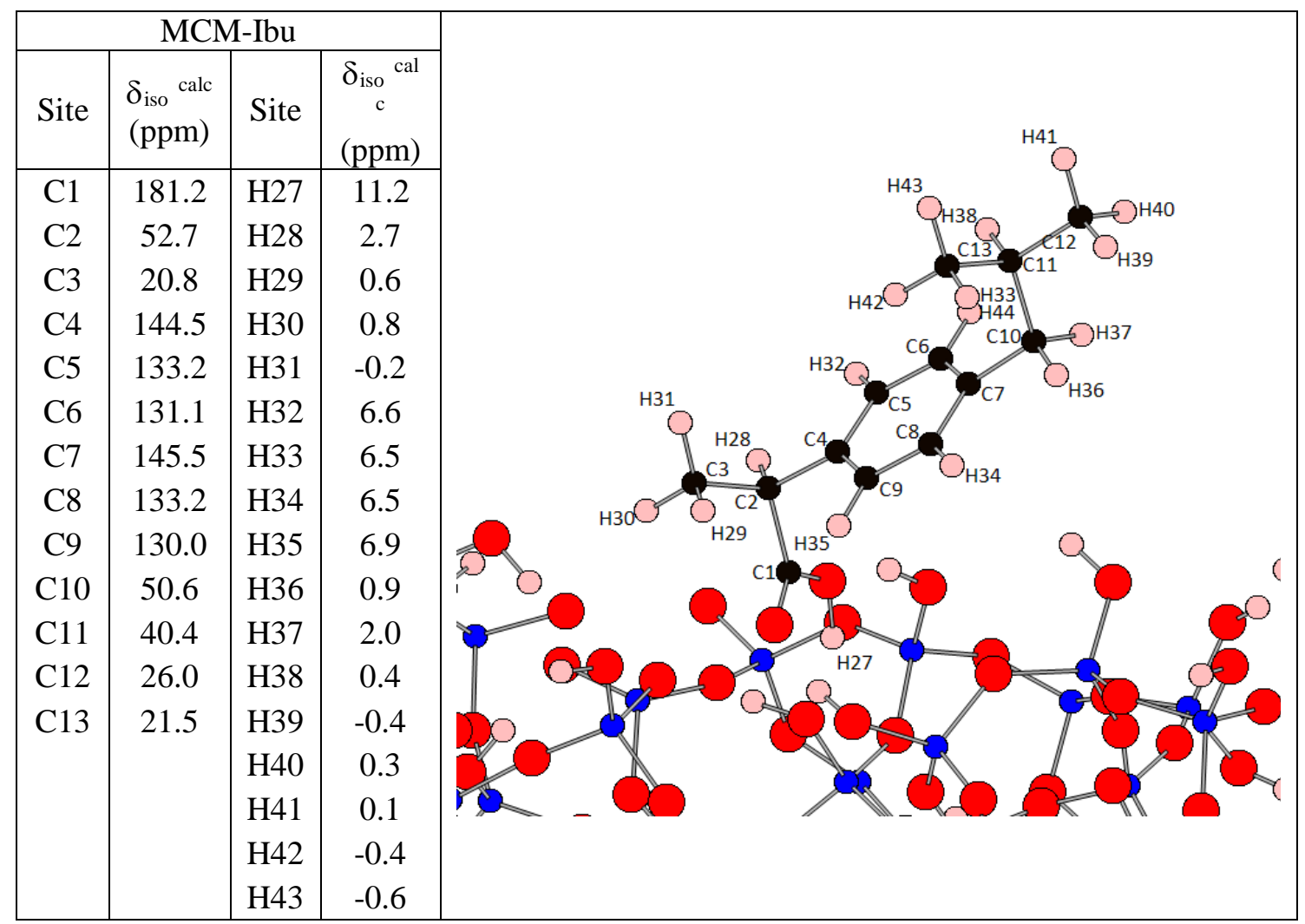


Table S6: Calculated ${ }^{13} \mathrm{C}$ and ${ }^{1} \mathrm{H}$ NMR chemical shift values for optimized geometries for Ibu adsorbed on micro-solvated hydroxylated amorphous silica

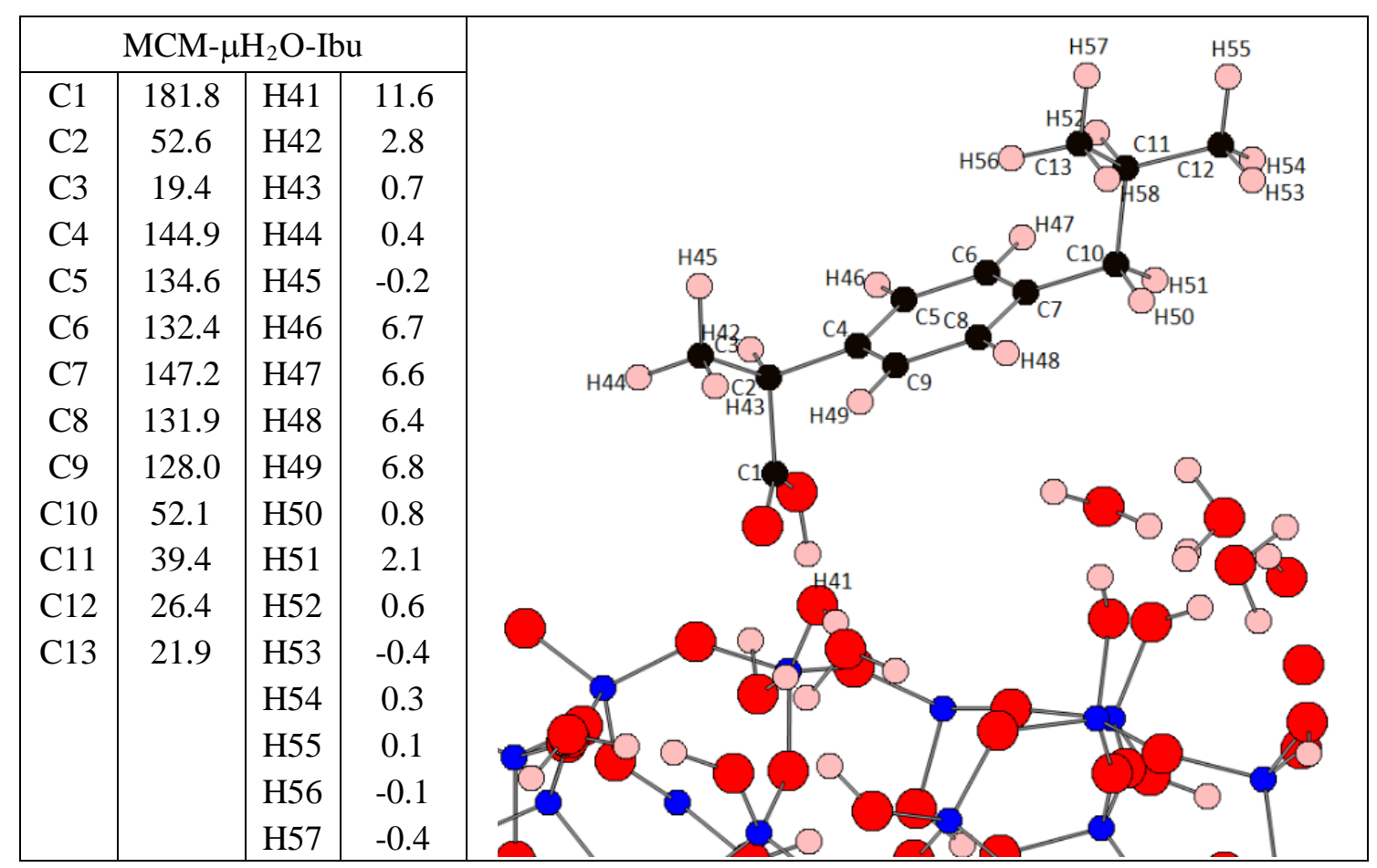


Table S7: Calculated ${ }^{13} \mathrm{C}$ and ${ }^{1} \mathrm{H}$ NMR chemical shift values for optimized geometries for Ibu adsorbed on functionalized hydroxylated amorphous silica.

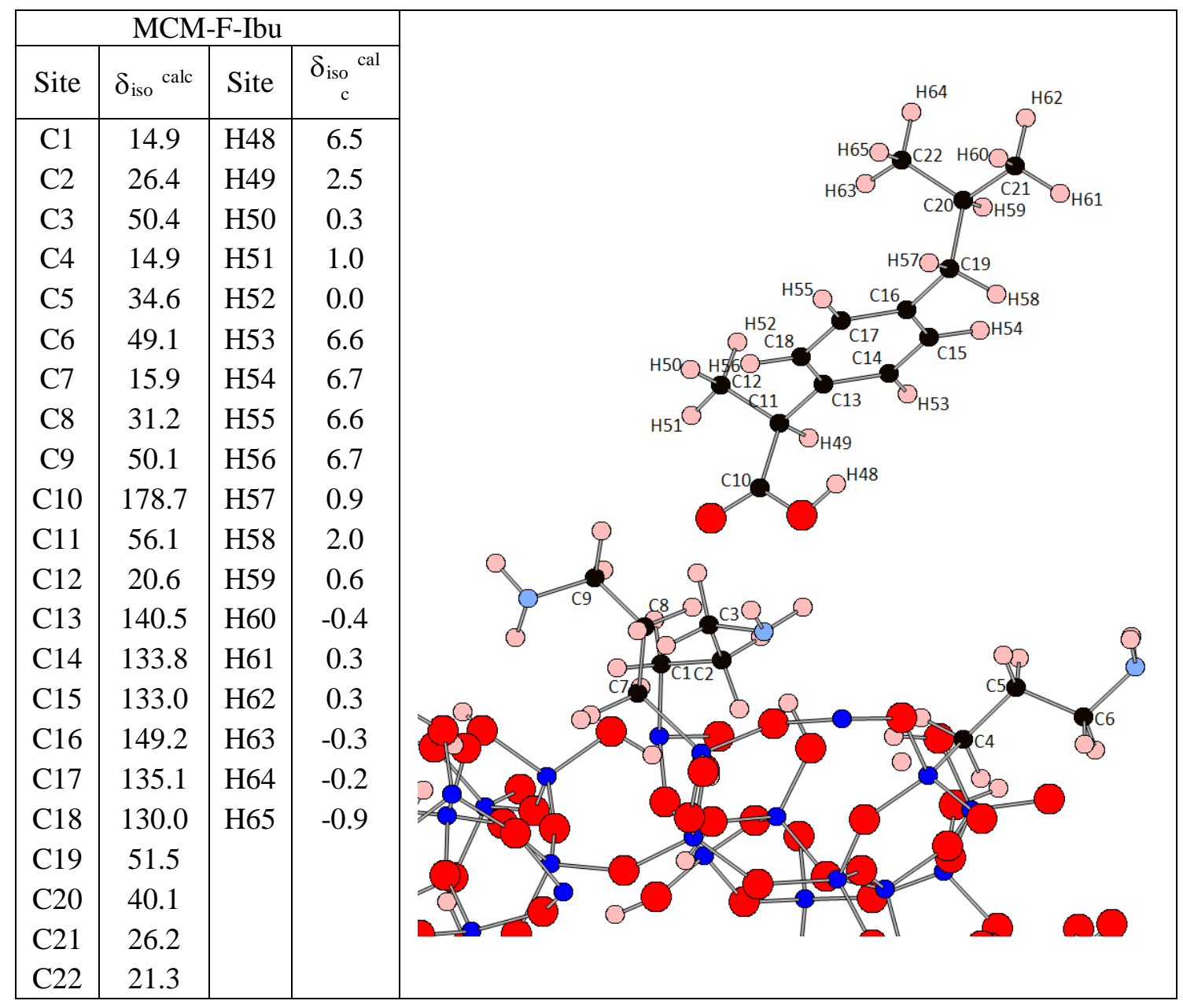


Table S8: Calculated ${ }^{13} \mathrm{C}$ and ${ }^{1} \mathrm{H}$ NMR chemical shift values for optimized geometries for Ibu adsorbed on micro-solvated functionalized hydroxylated amorphous silica.

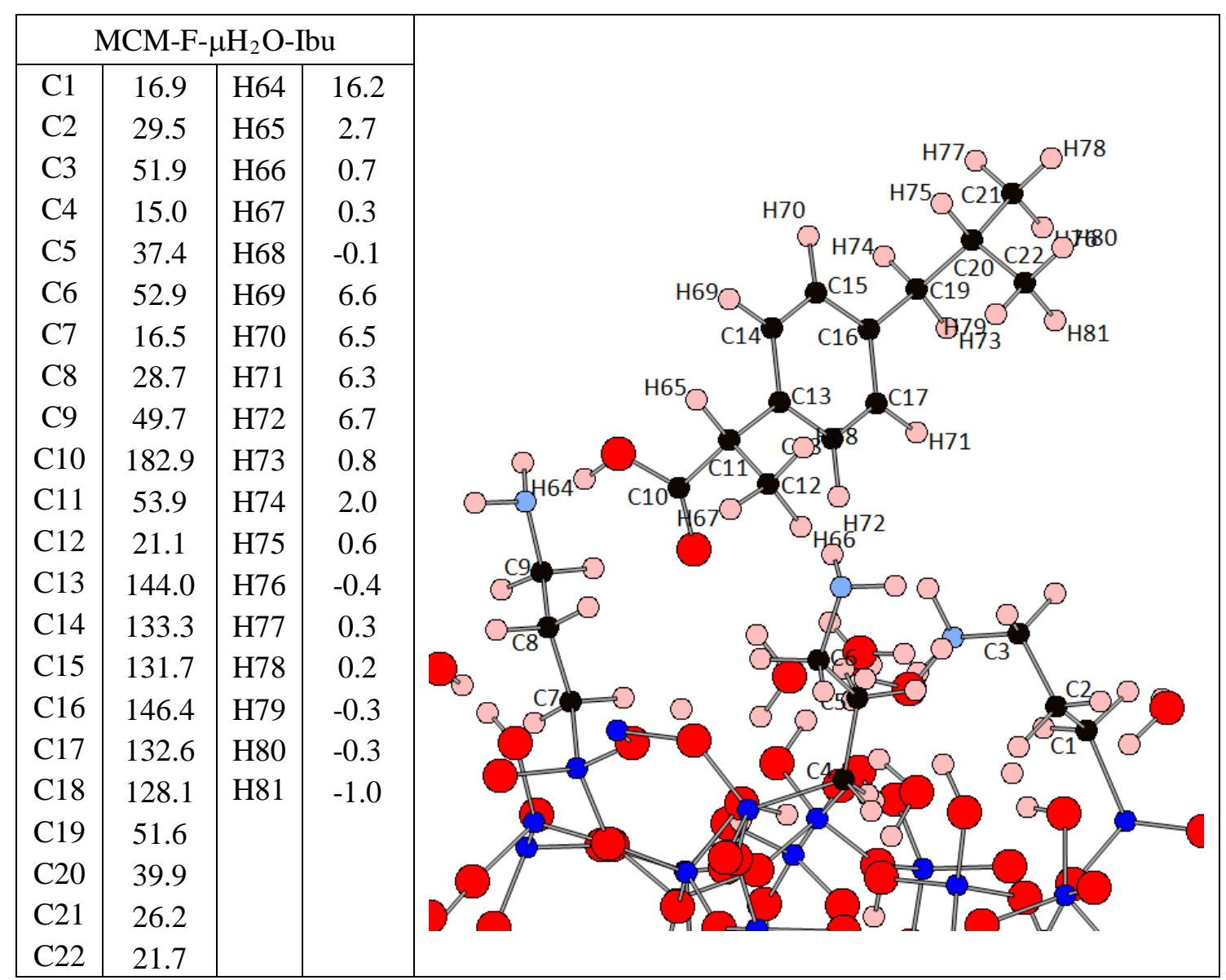

